\title{
Achieving Location Error Tolerant Barrier Coverage for Wireless Sensor Networks
}

\author{
Zhibo Wang ${ }^{\mathrm{a}}$, Honglong Chen ${ }^{\mathrm{b}}$, Qing $\mathrm{Cao}^{\mathrm{c}}$, Hairong Qi ${ }^{\mathrm{c}}$, Zhi Wang ${ }^{\mathrm{d}}$, Qian \\ Wang $^{\mathrm{a}}$ \\ ${ }^{a}$ State Key Lab of Software Engineering, School of Computer, Wuhan University, CHINA \\ ${ }^{b}$ Information and Control Engineering, China University of Petroleum, Qingdao, CHINA \\ ${ }^{c}$ Electrical Engineering and Computer Science, University of Tennessee, Knoxville, USA \\ ${ }^{d}$ State Key Laboratory of Industrial Control Technology, Hangzhou, CHINA
}

\begin{abstract}
Barrier coverage is a critical issue in wireless sensor networks deployed in security applications (e.g., border protection), whose performance strongly depends on the locations of sensor nodes. Existing works on barrier coverage typically assume that sensor nodes have accurate location information, which is not reasonable or practical for many real sensor networks. In this paper, we study the barrier coverage problem when sensor nodes have location errors and deploy mobile sensor nodes to improve barrier coverage if the network is not barriercovered after initial deployment. We analyze the effects of location errors for barrier coverage and propose a fault-tolerant weighted barrier graph to model the barrier coverage formation problem. Based on the graph, we prove that the minimum number of mobile sensor nodes needed to achieve barrier coverage with a guarantee is the length of the shortest path on the graph. Furthermore, we improve the computational efficiency of the fault-tolerant barrier coverage formation algorithm by removing unnecessary edges on the graph. Experimental results validate the correctness of our analysis and the proposed algorithms.
\end{abstract}

Keywords: Barrier coverage, location error, fault tolerance, sensor networks.

\section{Introduction}

Wireless sensor networks (WSNs) have been widely used as an effective surveillance tool for security applications, such as battlefield surveillance, border protection, and airport intruder detection. To detect intruders who penetrate the region of interest (ROI), we need to deploy a set of sensor nodes that can provide coverage of the ROI, a problem that is often referred to as barrier coverage [1], where sensor nodes form barriers for intruders.

Deterministic and random deployment are the two most popular ways of deploying sensor nodes in the ROIs. For ROIs within friendly environments, a

*Corresponding author: Qian Wang, qianwang@whu.edu.cn

Preprint submitted to Computer Networks

November 16, 2016

(C) 2016. This manuscript version is made available under the Elsevier user license http://www.elsevier.com/open-access/userlicense/1.0/ 
deterministic deployment can be used to deploy sensor nodes to specific locations. However, in general, the ROIs are within harsh environments that are difficult for humans to reach, which makes random deployment (e.g., dropping by aircraft) the only practical way to deploy nodes. When only stationary sensor nodes are used, after the initial random deployment, it is highly possible that sensor nodes could not form a barrier due to the gaps in their coverage, which would allow intruders to cross the ROIs without being detected. Therefore, it is necessary to deploy more sensor nodes to form a barrier. In fact, it is difficult, if possible at all, to improve barrier coverage for sensor networks consisting of only stationary nodes ${ }^{1}$. Fortunately, with recent technological advances, practical mobile nodes (e.g., Robomote [2] and Packbot [3]) have been developed, which provides us a way to improve barrier coverage performance after sensor networks have been deployed.

The location information of nodes provides the basis of many applications, such as navigation and target tracking. However, it is costly to equip each node with GPS receivers. Therefore, the locations of nodes are unknown when they are randomly deployed. To obtain the location of each node, a lot of localization algorithms have been proposed, including range-based (e.g., TOA [4], TDOA [5], and RSSI [6]) and range-free (e.g., DV-HOP [7][8], and APIT [9]) localization algorithms. However, none of them can provide accurate locations, which inevitably introduces location errors.

The existence of location errors can significantly affect the quality of barrier coverage provided by sensor networks. In reality, we can only know the measured locations instead of the true locations of sensor nodes. As shown in Figure 1(a), although nodes $a$ and $b$ actually overlap with each other ${ }^{2}$, due to the location errors, we think they do not, and need to deploy more mobile nodes between them to prevent intruders from crossing without being detected, which increases the cost of deployment. In contrast, as shown in Figure 1(b), based on the measured locations, we think nodes $a$ and $b$ overlap with each other and all intruders crossing the line segment $a b$ can be detected. However, since they actually do not overlap, intruders can cross the line segment $a b$ without being detected. Therefore, location errors cannot only increase the cost of node deployment but also increase the miss rate of intruders.

A lot of work has been done on barrier coverage. However, few works consider the effects of location errors of sensor nodes [10]. Shen et al. only considered the line-based barrier coverage formation problem where nodes have location errors in the $x$-axis and did not consider the sensor movement problem [10]. In this paper, we explore the effects of location errors on barrier coverage on a two-dimensional plane and study how to leverage mobile nodes to form a barrier with certainty when nodes have location errors.

The topic studied in this paper involves two main problems. First, how

\footnotetext{
${ }^{1}$ We use "sensor nodes" or "nodes" interchangeably in this paper.

${ }^{2}$ In this paper, "nodes overlap with each other" means "the sensing regions of nodes overlap with each other".
} 


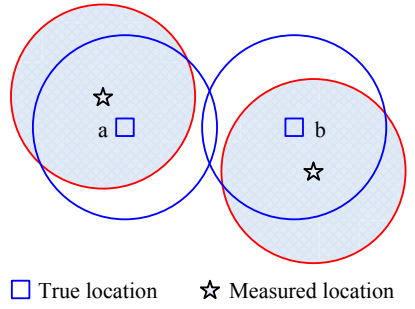

(a) overlap $\rightarrow$ no overlap

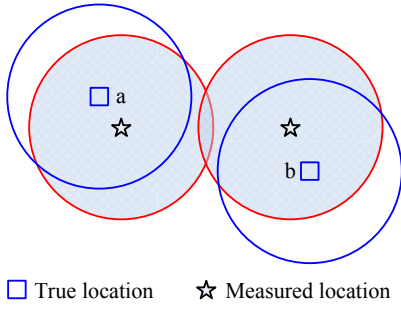

(b) no overlap $\rightarrow$ overlap

Figure 1: The effects of location errors. According to the measured locations, (a): overlapping nodes are considered as no overlapping; (b): no overlapping nodes are considered as overlapping

can we know whether the network provides barrier coverage or not after initial random deployment when nodes have location errors? Even if, based on the measured locations, the network provides barrier coverage for the ROI, in reality it may not. When the ROI is not barrier-covered, mobile nodes can be further deployed to form barrier coverage. Note that the manufacturing cost of mobile nodes is usually much higher than stationary nodes, which demands the usage of as few mobile nodes as possible. Therefore, the second problem that must be solved is to find the minimum number of mobile nodes needed to form barrier coverage when nodes have location errors. The problem is challenging since the minimum number of mobile nodes needed to connect a pair of two stationary nodes calculated from the distance between measured locations instead of true locations may not be enough in reality. Moreover, there are too many holes in the network and there are numerous ways of filling in different sets of holes to form barrier coverage. Thus, it is challenging to find the optimal way that uses the minimum number of mobile nodes to form a barrier with stationary nodes with a guarantee.

In this paper, we address these problems and propose a fault-tolerant barrier coverage formation problem to form a barrier with a guarantee when nodes have location errors. To the best of our knowledge, this work makes the first attempt exploring the effects of location errors on barrier coverage. Without the study of location errors, any solutions to the barrier coverage problem would only be limited to theoretical study and cannot be applied in practice, as in the real world location errors are ubiquitous. The main contributions are summarized as follows.

- We analyze the effects of location errors on the minimum number of mobile nodes needed to fill in a gap when only stationary nodes and both stationary and mobile nodes have location errors.

- We propose a fault-tolerant weighted barrier graph to model the barrier coverage formation problem, and prove that the minimum number of mobile nodes needed to achieve barrier coverage with a guarantee is the length of the shortest path on the graph. 
- We propose a fault-tolerant barrier coverage algorithm for the cases where nodes have location errors, and further improve the computational efficiency of the proposed algorithm by removing unnecessary edges on the weighted barrier graph.

The remainder of the paper is organized as follows. We discuss the literature of barrier coverage in Section 2. We present the system model in Section 3. We study the barrier coverage problem when only stationary nodes have location errors in Section 4 and the barrier coverage problem when both stationary and mobile nodes have location errors in Section 5. The algorithm analysis and improvement are described in Section 6. The performance evaluation is presented in Section 7. Finally, we conclude the paper in Section 8.

\section{Related Work}

Kumar et al. first introduced two notions of probabilistic barrier coverage - weak barrier coverage and strong barrier coverage [1]. The former requires the union of nodes to form a barrier such that nodes crossing the ROI with a fixed, constant direction will be detected, while the latter requires the union of nodes to form a barrier so that every intruder can be detected no matter what crossing path it takes. Liu et al. [11] devised an efficient distributed algorithm to construct multiple disjoint barriers for strong barrier coverage in a randomly deployed sensor network on a long irregular strip region. He et al. [12] studied the optimal node deployment for curve-based barrier coverage. Wang et al. explored the optimal node deployment for belt barrier coverage $[13,14]$ and perimeter barrier coverage [15]. Kim et al. proposed a new type of barrier, $k$-event-driven partial barriers, with static sensor nodes in [16] and further introduced resilient event-driven partial barriers with mobile sensors in [17]. Wang et al. summarized the coverage problems in sensor networks [18], and Wu et al. summarized the works on barrier coverage with nodes in [19].

With the development of mobile sensors, sensor mobility is exploited to improve barrier coverage. Shen et al. [20] studied the energy-efficient relocation problem for barrier coverage in mobile sensor networks. Keung et al. [21] focused on providing $k$-barrier coverage against moving intruders in mobile sensor networks. Kong et al. [22] studied the problem of using mobile sensors to form a barrier surrounding a region. Chen et al. [23] studied the problem moving $n$ sensors on a line to form a barrier on a specified segment of the line such that the maximum moving distance of the sensors is minimized. Silvestri et al. [24] proposed MobiBar, a distributed and asynchronous algorithm for $k$ barrier coverage formation with mobile sensors. Fault-tolerant event detection and line-based barrier coverage formation algorithm in mobile WSNs were studied in [10][25]. He et al. [26] studied the cost-effective barrier coverage problem when there are not sufficient mobile sensors. Recently, the authors also studied the area coverage problem in WSNs [27][28].

Tian et al. [29] studied the barrier coverage problem in a mobile survivabilityheterogeneous WSNs and proposed a grid-based greedy barrier construction 
algorithm to construct a strong barrier. Saipulla et al. [30] found the gaps between stationary sensors and relocated mobile sensors to form a barrier while balancing the energy consumption among mobile sensors, and further studied how to relocate mobile sensors if their mobility ability is limited [31]. In [32], the authors studied how to minimize the cost of forming $k$-barrier coverage with predeployed stationary sensors. They proposed a weighted barrier graph to model the barrier formation problem and proved that the minimum number of mobile nodes needed to form $k$-barrier coverage is related to the $k$ vertex-disjoint paths with the minimum total length on the graph. Zhang et al. relocated mobile nodes to achieve $k$-barrier coverage based on integer linear programming [33].

Much work has been done on barrier coverage; however, authors always assume that the locations of nodes are known and accurate, which is not reasonable or practical for most real sensor networks since location errors are ubiquitous in the real world. In this paper, we analyze the effects of location errors on barrier coverage and propose an efficient fault-tolerant barrier coverage algorithm to form a barrier with a guarantee when nodes have location errors.

The preliminary results of this paper have been published in [34]. In this paper, we further analyze the computational complexity of the proposed faulttolerant barrier coverage algorithm, and propose an improved algorithm to reduce its computational cost. Moreover, we relax the assumption of location errors for mobile nodes and derive the effects of location errors of mobile nodes on barrier coverage.

\section{System Model}

We assume that the ROI is a two-dimensional rectangular belt area and $n$ stationary sensor nodes are randomly deployed in it. The belt region with the length of $L$ and the width of $H$ is generally a long and thin strip. There is a base station in the ROI which has all the nodes' measured locations. In this paper, we mainly focus on studying the effects of location errors on barrier coverage and do not consider the communication issues of sensor nodes. An intruder may attempt to penetrate the area along any crossing path.

We assume that stationary and mobile sensor nodes have the same type of sensors, but mobile sensor nodes have the ability to move. We adopt the most commonly used disk model for the sensing ability of sensor nodes, and assume that all sensor nodes have the same sensing range, denoted by $r_{s}$. That is, when an intruder is within the distance of $r_{s}$ of a sensor node, the sensor can detect the intruder; otherwise, the sensor cannot detect the intruder. Let $s_{i}$ denote the $i$-th sensor node whose true location is $l_{i}=\left(x_{i}, y_{i}\right)$. Each node can obtain an estimate of its location, denoted by $\tilde{l}_{i}=\left(\tilde{x}_{i}, \tilde{y}_{i}\right)$ for $s_{i}$. Thus, $d\left(l_{i}, \tilde{l}_{i}\right)$ is called the location error for $s_{i}$, where $d(\cdot)$ represents the Euclidean distance. We assume that the location error is upper bounded by $\delta$ where $\delta<r_{s}$. The notations used throughout the paper are summarized in Table 1. 
Table 1: Summary of notations

\begin{tabular}{c|l}
\hline Symbol & Description \\
\hline$L$ & the length of the belt region \\
$H$ & the width of the belt region \\
$n$ & the number of deployed stationary nodes \\
$r_{s}$ & the sensing range of each sensor node \\
$\delta$ & the upper bound of location errors of stationary nodes and $\delta<r_{s}$ \\
$\delta^{\prime}$ & the upper bound of location errors of mobile nodes and $\delta^{\prime} \leq \delta$ \\
$s_{i}$ & the $i$ th stationary sensor node \\
$l_{i}$ & $l_{i}=\left(x_{i}, y_{i}\right)$ the true location of $s_{i}$ \\
$\tilde{l}_{i}$ & $\tilde{l}_{i}=\left(\tilde{x}_{i}, \tilde{y}_{i}\right)$ the measured location of $s_{i}$ \\
$R_{i}$ & Location region of $s_{i}$ \\
$d\left(l_{i}, l_{j}\right)$ & the true distance between $s_{i}$ and $s_{j}$ \\
$d\left(\tilde{l}_{i}, \tilde{l}_{j}\right)$ & the measured distance between $s_{i}$ and $s_{j}$ \\
$N\left(s_{i}, s_{j}\right)$ & the true number of mobile nodes needed to connect $s_{i}$ and $s_{j}$ \\
$N_{s}^{u}\left(s_{i}, s_{j}\right)$ & the upper bound of $N\left(s_{i}, s_{j}\right)$ when stationary nodes have location errors \\
$N_{s}^{l}\left(s_{i}, s_{j}\right)$ & the lower bound of $N\left(s_{i}, s_{j}\right)$ when stationary nodes have location errors \\
$N_{s m}^{u}\left(s_{i}, s_{j}\right)$ & the upper bound of $N\left(s_{i}, s_{j}\right)$ when both stationary and mobile nodes have \\
$N_{s m}^{l}\left(s_{i}, s_{j}\right)$ & location errors \\
& the lower bound of $N\left(s_{i}, s_{j}\right)$ when both stationary and mobile nodes have \\
\hline
\end{tabular}

\section{Barrier Coverage When Stationary Nodes have Location Errors}

In this section, we assume that only stationary nodes have location errors. We assume that mobile nodes are equipped with GPS receivers, so that they can accurately know their locations without errors.

\subsection{Location Error Analysis}

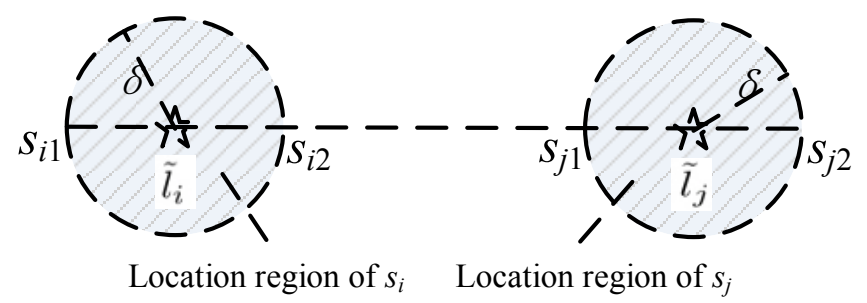

Figure 2: The location region of a sensor node given its measured location

The basis of barrier coverage is to decide whether two nodes overlap or not and how many mobile nodes are needed when they do not overlap. The problem is easy to answer if each node knows its true location. For example, given two nodes $s_{i}$ and $s_{j}$ and their true locations $l_{i}$ and $l_{j}$, they overlap with each other if $d\left(l_{i}, l_{j}\right) \leq 2 r_{s}$. When $d\left(l_{i}, l_{j}\right)>2 r_{s}, s_{1}$ and $s_{2}$ do not overlap with each other and the exactly number of mobile nodes needed to connect them, denoted by $N\left(s_{i}, s_{j}\right)$, is $\left\lceil\frac{d\left(l_{i}, l_{j}\right)-2 r_{s}}{2 r_{s}}\right\rceil$.

However, the problem is that each node does not know its true location but instead its measured location. Suppose the measured locations for $s_{i}$ and $s_{j}$ are 
$\tilde{l}_{i}$ and $\tilde{l}_{j}$, respectively. As shown in Figure 2, given a measured location, the true location is within the shaded circle centered at the measured location with a radius of $\delta$, where $\delta$ is the upper bound of location errors. We call the shaded circle centered at $\tilde{l}_{i}$ as the location region of $s_{i}$, denoted by $R_{i}$. Therefore,

$$
\max \left(0, d\left(\tilde{l}_{i}, \tilde{l}_{j}\right)-2 \delta\right) \leq d\left(l_{i}, l_{j}\right) \leq d\left(\tilde{l}_{i}, \tilde{l}_{j}\right)+2 \delta .
$$

Given two stationary nodes $s_{i}$ and $s_{j}$, and their measured locations $\tilde{l}_{i}$ and $\tilde{l_{j}}$, when $d\left(\tilde{l}_{i}, \tilde{l}_{j}\right)+2 \delta \leq 2 r_{s}$, the true distance $d\left(l_{i}, l_{j}\right) \leq 2 r_{s}$. Therefore, $s_{i}$ and $s_{j}$ overlap with each other with a guarantee when $d\left(\tilde{l}_{i}, \tilde{l}_{j}\right)+2 \delta \leq 2 r_{s}$.

Lemma 1. Given two stationary nodes $s_{i}$ and $s_{j}$, and their measured locations $\tilde{l}_{i}$ and $\tilde{l}_{j}$, when $d\left(\tilde{l}_{i}, \tilde{l}_{j}\right)>2 r_{s}-2 \delta$, the minimum number of mobile nodes needed to guarantee the connection of $s_{i}$ and $s_{j}$ is $\left\lceil\frac{d\left(\tilde{l}_{i}, \tilde{l}_{j}\right)+2 \delta}{2 r_{s}}\right\rceil-1$.

Proof. Recall that $N\left(s_{i}, s_{j}\right)=\left\lceil\frac{d\left(l_{i}, l_{j}\right)-2 r_{s}}{2 r_{s}}\right\rceil$ denotes the true number of mobile nodes needed to connect $s_{i}$ and $s_{j}$. Since $\max \left(0, d\left(\tilde{l}_{i}, \tilde{l}_{j}\right)-2 \delta\right) \leq d\left(l_{i}, l_{j}\right) \leq$ $d\left(\tilde{l}_{i}, \tilde{l}_{j}\right)+2 \delta$, we have

$$
\max \left(0,\left\lceil\frac{d\left(\tilde{l}_{i}, \tilde{l}_{j}\right)-2 \delta}{2 r_{s}}\right\rceil-1\right) \leq N\left(s_{i}, s_{j}\right) \leq\left\lceil\frac{d\left(\tilde{l}_{i}, \tilde{l}_{j}\right)+2 \delta}{2 r_{s}}\right\rceil-1 .
$$

In order to guarantee the connection of $s_{i}$ and $s_{j}$, the upper bound of $N\left(s_{i}, s_{j}\right)$, $\left\lceil\frac{d\left(\tilde{l}_{i}, \tilde{l}_{j}\right)+2 \delta}{2 r_{s}}\right\rceil-1$, mobile nodes are needed.

Let $N_{s}^{u}\left(s_{i}, s_{j}\right)=\left\lceil\frac{d\left(\tilde{l}_{i}, \tilde{l}_{j}\right)+2 \delta}{2 r_{s}}\right\rceil-1$ denote the upper bound of $N\left(s_{i}, s_{j}\right)$, and $N_{s}^{l}\left(s_{i}, s_{j}\right)=\max \left(0,\left\lceil\frac{d\left(\tilde{l}_{i}, \tilde{l}_{j}\right)-2 \delta}{2 r_{s}}\right\rceil-1\right)$ denote the lower bound of $N\left(s_{i}, s_{j}\right)$. Thus, $\triangle N_{s}\left(s_{i}, s_{j}\right)=N_{s}^{u}\left(s_{i}, s_{j}\right)-N_{s}^{l}\left(s_{i}, s_{j}\right)$ represents the effects of location errors on the minimum number of mobile nodes needed to connect $s_{i}$ and $s_{j}$. When $\triangle N_{s}\left(s_{i}, s_{j}\right)=0, N\left(s_{i}, s_{j}\right)=N_{s}^{u}\left(s_{i}, s_{j}\right)=N_{s}^{l}\left(s_{i}, s_{j}\right)$ and the location errors would not affect the minimum number of mobile nodes needed to connect $s_{i}$ and $s_{j}$.

Lemma 2. Given two stationary nodes $s_{i}$ and $s_{j}$, and their measured locations $\tilde{l}_{i}$ and $\tilde{l}_{j}$, the location error does not affect the minimum number of mobile nodes needed to connect $s_{i}$ and $s_{j}$ when either $d\left(\tilde{l}_{i}, \tilde{l}_{j}\right)+2 \delta \leq 2 r_{s}$ or $\left\lceil\frac{d\left(\tilde{l}_{i}, \tilde{l}_{j}\right)-2 \delta}{2 r_{s}}\right\rceil=$ $\left\lceil\frac{d\left(\tilde{l}_{i}, \tilde{l}_{j}\right)+2 \delta}{2 r_{s}}\right\rceil \geq 2$.

Proof. When $N_{s}^{u}\left(s_{i}, s_{j}\right)=N_{s}^{l}\left(s_{i}, s_{j}\right)=0,\left\lceil\frac{d\left(\tilde{l}_{i}, \tilde{l}_{j}\right)+2 \delta}{2 r_{s}}\right\rceil-1$ should be 0 . Therefore, $d\left(\tilde{l}_{i}, \tilde{l}_{j}\right)+2 \delta \leq 2 r_{s}$ is required.

When $N_{s}^{u}\left(s_{i}, s_{j}\right)=\left\lceil\frac{d\left(\tilde{l}_{i}, \tilde{l}_{j}\right)+2 \delta}{2 r_{s}}\right\rceil-1=N_{s}^{l}\left(s_{i}, s_{j}\right)=\left\lceil\frac{d\left(\tilde{l}_{i}, \tilde{l}_{j}\right)-2 \delta}{2 r_{s}}\right\rceil-1>0$, $\left\lceil\frac{d\left(\tilde{l}_{i}, \tilde{l}_{j}\right)-2 \delta}{2 r_{s}}\right\rceil=\left\lceil\frac{d\left(\tilde{l}_{i}, \tilde{l}_{j}\right)+2 \delta}{2 r_{s}}\right\rceil=k$ is required where $k$ is an integer and $k \geq 2$.

Therefore, when any one of them is satisfied, the location error does not affect the minimum number of mobile nodes needed to connect $s_{i}$ and $s_{j}$. 
Theorem 3. Given a sensor network where only stationary nodes have location errors upper bounded by $\delta<r_{s}$, for any pair of stationary nodes, at most 2 more mobile nodes are needed to connect the pair of stationary nodes compared to the true minimum number of mobile sensor nodes needed. That is, $\triangle N_{s}\left(s_{i}, s_{j}\right) \leq 2$ for any pair of $s_{i}$ and $s_{j}$ when $\delta<r_{s}$.

Proof. See the proof in Appendix A.

\subsection{Progressive Mobile Node Deployment}

For any two known true locations of $s_{i}$ and $s_{j}$ within $R_{i}$ and $R_{j}$ respectively, $N_{s}^{u}\left(s_{i}, s_{j}\right)$ is enough to connect them with a guarantee. However, the difficulty is that the true locations are unknown in reality, so the problem is how to deploy $N_{s}^{u}\left(s_{i}, s_{j}\right)$ mobile nodes to connect $s_{i}$ and $s_{j}$ with a guarantee. We propose a progressive deployment method to solve this problem.

The basic idea of the progressive method is to deploy mobile nodes progressively from the left stationary node to the right stationary node. Given two stationary nodes $s_{i}$ and $s_{j}$, and their measured locations $\tilde{l}_{i}$ and $\tilde{l}_{j}$, the progressive method is described as follows:

- Step 1: Deploy a mobile node on the line segment $\tilde{l}_{i} \tilde{l}_{j}$ to make it overlap with all nodes located within the location region of $s_{i}$ and the distance between the mobile node and $\tilde{l}_{i}$ maximized.

- Step 2: Check whether the new deployed mobile node overlaps with all nodes located within the location region of $s_{j}$ or not. If yes, stop; otherwise, go to step 3 .

- Step 3: Deploy a new mobile node on the line segment $\tilde{l}_{i} \tilde{l}_{j}$ that is $2 r_{s}$ away from the previously deployed mobile node, go to step 2 .

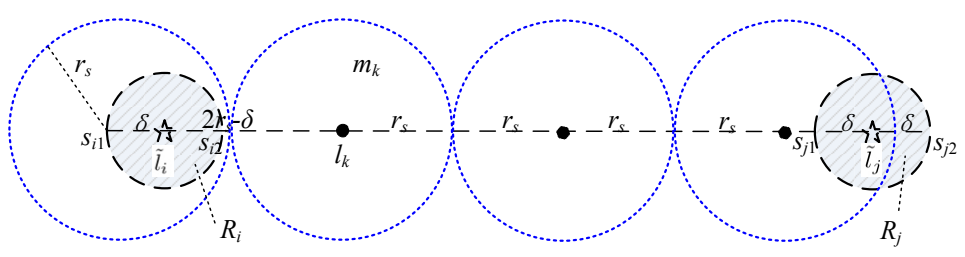

is Measured location - Expected location for mobile sensor node

Figure 3: An illustration of the progressive method. The blue solid circle with radius of $r_{s}$ denotes the sensing region of $s_{i}$ located at $s_{i 1}$. The blue dashed circles with radius of $r_{s}$ denote the sensing regions of mobile nodes.

Figure 3 shows an example of the progressive deployment method. $s_{i 1}$ and $s_{i 2}$ are the two intersections of line $\tilde{l}_{i} \tilde{l}_{j}$ and the location region of $s_{i}$. For any point $p$ on $\tilde{l}_{i} \tilde{l}_{j}$, the largest distance from $p$ to any point within $R_{i}$ is the distance from $p$ to $s_{i 1}$. In other words, if the mobile node at point $l_{k}$ overlaps with a node at point $s_{i 1}$, it overlaps all the nodes located within $R_{i}$. As $m_{k}$ moves along $\tilde{l}_{i} \tilde{l}_{j}$, both $d\left(s_{i 1}, l_{k}\right)$ and $d\left(\tilde{l}_{i}, l_{k}\right)$ increase accordingly. When $d\left(\tilde{l}_{i}, l_{k}\right)=2 r_{s}-\delta$, 
$m_{k}$ cannot move further since any further movement would not guarantee the overlap of $m_{k}$ and $s_{i}$. Therefore, the maximum of $d\left(\tilde{l_{i}}, l_{k}\right)$ is $2 r_{s}-\delta$. When more mobile nodes are required, they will be added one by one with the interval of $2 r_{s}$ until the distance between a mobile node and $s_{j 2}$ is no larger than $2 r_{s}$. Therefore, the number of mobile nodes needed in the progressive method is $\left\lceil\frac{d\left(\tilde{l}_{i}, \tilde{l}_{j}\right)+2 \delta}{2 r_{s}}\right\rceil-1$. That is, the progressive method is an optimal deployment strategy that connects $s_{i}$ and $s_{j}$ with a guarantee by using $\left\lceil\frac{d\left(\tilde{l}_{i}, \tilde{l}_{j}\right)+2 \delta}{2 r_{s}}\right\rceil-1$ mobile nodes.

\subsection{Fault-tolerant Barrier Coverage Formation}

Mobile nodes can be deployed between stationary nodes to fill in gaps to form a barrier. However, there are too many ways to deploy mobile nodes and how to find the optimal way using the minimum number of mobile nodes is challenging. In this subsection, we adopt the barrier graph model of [32] and propose a fault-tolerant weighted barrier graph to model the barrier coverage formation problem with location errors and prove that the minimum number of mobile nodes needed to form barrier coverage with a guarantee is the length of the shortest path on the graph.

Definition 1. A fault-tolerant weighted barrier graph (FTWBG) $G=$ $(V, E, W)$ of a sensor network is constructed as follows. The set $V$ consists of vertices corresponding to the left boundary $(s)$, all the stationary sensors $(S)$ and the right boundary $(t)$ of the belt region, that is, $V=\left\{v_{1}, v_{2}, \ldots, v_{n+2}\right\}=$ $\{s\} \cup S \cup\{t\} . E=\left\{e\left(v_{i}, v_{j}\right)\right\}$ is the set of edges between any pair of vertices. $W: E \rightarrow \mathbb{R}$ is the set of weights of each edge, where the weight $w\left(v_{i}, v_{j}\right)$ of edge $e\left(v_{i}, v_{j}\right)$ is the minimum number of mobile nodes needed to guarantee the connection of $v_{i}$ and $v_{j}$.

In order to guarantee the connection of $s_{i}$ and $s_{j},\left\lceil\frac{d\left(\tilde{l}_{i}, \tilde{l}_{j}\right)+2 \delta}{2 r_{s}}\right\rceil-1$ mobile nodes should be deployed and therefore $w\left(s_{i}, s_{j}\right)=\left\lceil\frac{d\left(\tilde{l}_{i}, \tilde{l}_{j}\right)+2 \delta}{2 r_{s}}\right\rceil-1$. For a node $s_{j}$, the maximum distance between that node and the left boundary $s$ is $\tilde{x}_{j}+\delta$, where $\tilde{x}_{j}$ is the $x$-coordinate of the measured location of $s_{j}$. In order to guarantee the connection of them, $w\left(s, s_{j}\right)=\left\lceil\frac{\tilde{x}_{j}+\delta-r_{s}}{2 r_{s}}\right\rceil$ mobile nodes are needed. Also, the maximum distance between $s_{j}$ and the right boundary $t$ is $L-$ $\left(\tilde{x}_{j}-\delta\right)$. In order to guarantee the connection of them, $w\left(t, s_{j}\right)=\left\lceil\frac{L-\left(\tilde{x}_{j}-\delta+r_{s}\right)}{2 r_{s}}\right\rceil$ mobile nodes are needed. We can also deploy mobile nodes directly from the left boundary to the right boundary, and the minimum number of mobile nodes needed to connect $s$ and $t$ is $w(s, t)=\left\lceil\frac{L}{2 r_{s}}\right\rceil$. In summary, we have

$$
w\left(v_{i}, v_{j}\right)= \begin{cases}\left\lceil\frac{d\left(\tilde{l}_{i}, \tilde{l}_{j}\right)+2 \delta}{2 r_{s}}\right\rceil-1, & \text { if } v_{i}=s_{i} \text { and } v_{j}=s_{j} \\ \left\lceil\frac{\tilde{x}_{j}+\delta-r_{s}}{2 r_{s}}\right\rceil, & \text { if } v_{i}=s \text { and } v_{j}=s_{j} \\ \left\lceil\frac{L-\left(\tilde{x}_{j}-\delta+r_{s}\right)}{2 r_{s}}\right\rceil, & \text { if } v_{i}=t \text { and } v_{j}=s_{j} \\ \left\lceil\frac{L}{2 r_{s}}\right\rceil, & \text { if } v_{i}=s \text { and } v_{j}=t\end{cases}
$$


Figure 4 shows a deployed sensor network and its corresponding FTWBG. $s$ and $t$ are the virtual vertices corresponding to the left and right boundary of the belt region, respectively. The weight of each edge is the minimum number of mobile sensor nodes needed to guarantee the connection of the pair of vertices.

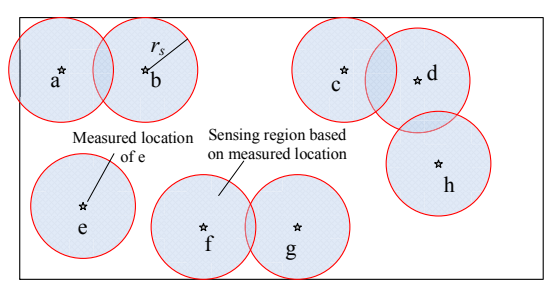

(a) Deployed sensor network

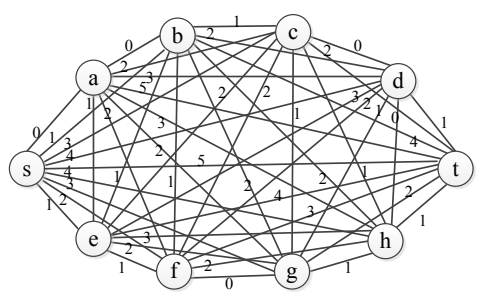

(b) Fault-tolerant weighted barrier graph

Figure 4: A sensor network and its corresponding FTWBG when only stationary nodes have location errors $\left(r_{s}=10 \mathrm{~m}\right.$ and $\left.\delta=1 \mathrm{~m}\right)$

Theorem 4. The minimum number of mobile nodes needed to form a barrier with a guarantee with stationary nodes is exactly the length of the shortest path from s to $t$ on the FTWBG $G$ and upper bounded by $\left\lceil\frac{L}{2 r_{s}}\right\rceil$.

Proof. According to the definition of the FTWBG $G$, if we want to form a barrier, we only need to choose a path from $s$ to $t$, and put exactly the number of mobile nodes needed on each edge of the path. That is, for a chosen path, the number of mobile nodes required to form a barrier with a guarantee is equal to the sum of weights of all edges on the path, which is the length of the path. Therefore, the minimum number of mobile nodes required to form a barrier with a guarantee is the length of the shortest path from $s$ to $t$ on graph $G$.

The path containing only the edge $e(s, t)$ could either be the shortest or not. If it is not the shortest path, the minimum number of mobile nodes required is smaller than $w(s, t)$; otherwise, the minimum number of mobile nodes required is equal to $w(s, t)$. Therefore, the minimum number of mobile nodes required to form a barrier with a guarantee is always upper bounded by $w(s, t)=\left\lceil\frac{L}{2 r_{s}}\right\rceil$.

Corollary 5. The ROI is guaranteed to be barrier-covered after initial deployment of nodes if and only if the length of the shortest path from $s$ to $t$ on $G$ equals zero.

Proof. The proof is omitted as it is straightforward.

According to Theorem 4, we can use the classic Dijkstra's algorithm [35] to find the minimum number of mobile nodes needed to form barrier coverage with a guarantee and check whether the ROI is guaranteed to be barrier-covered or not after initial deployment. As shown in Figure 4, the shortest path is $s \rightarrow a \rightarrow b \rightarrow c \rightarrow d \rightarrow t$, the length of which is $0+0+1+0+1=2$. Therefore, the ROI is not guaranteed to be barrier-covered after initial random deployment 
and 2 mobile nodes are needed to deploy between $b$ and $c$, and between $d$ and the right boundary to guarantee the formation of barrier coverage.

After the required number is calculated, mobile nodes are randomly deployed in the network and should move to fill in the gaps on the shortest path to form a barrier. In order to prolong the lifetime of mobile nodes, the total moving distance should be minimized. We formulate the problem as a minimum cost bipartite assignment problem in [32] and use the Hungarian algorithm [36] to find the optimal assignment strategy for mobile nodes to different gaps.

Based on the aforementioned analyses, we propose a fault-tolerant barrier coverage algorithm (FatBaCa) to find the minimum number of mobile nodes needed to form a barrier when nodes have location errors, which is formally described in Algorithm 1.

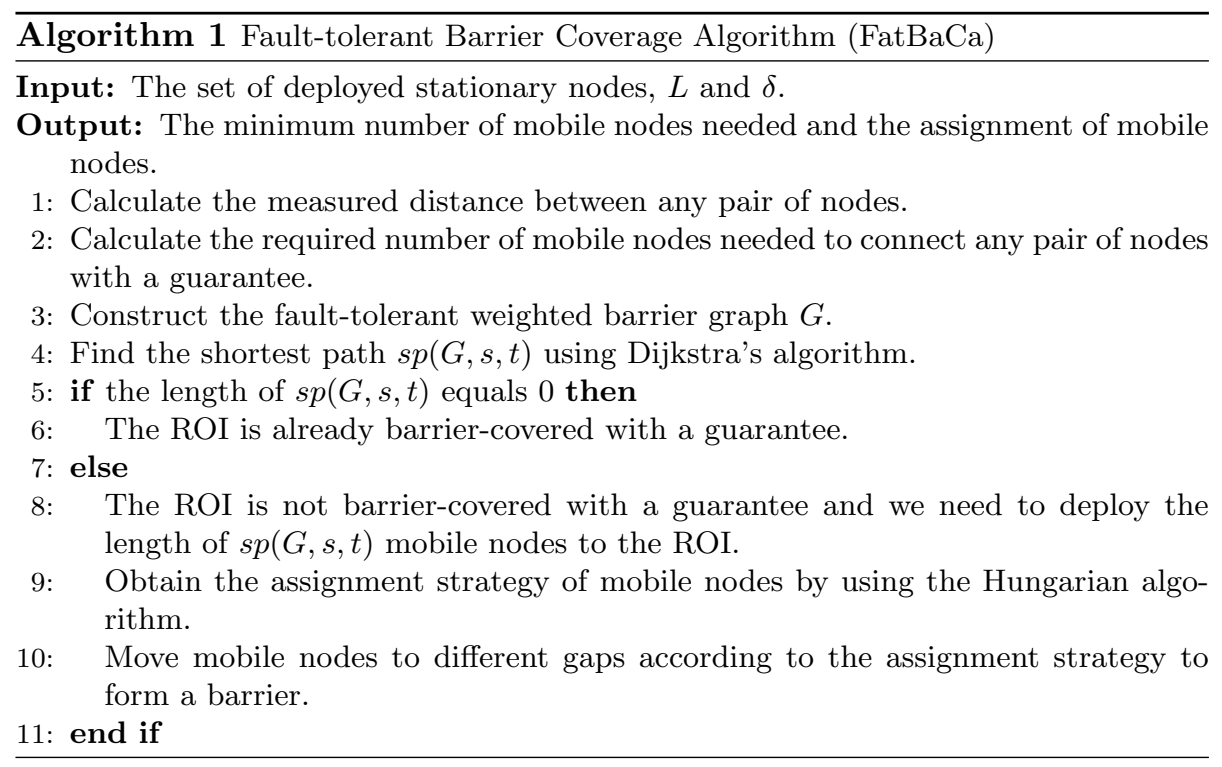

\section{Barrier Coverage When both Stationary and Mobile Nodes have Location Errors}

When the accuracy of GPS equipment on the mobile nodes is not very high, or mobile nodes are localized by using the localization algorithm also used by the stationary nodes, mobile nodes also suffer from location errors. In this section, we consider the situation that not only stationary nodes but also mobile nodes have location errors. We assume the location errors of mobile nodes are upper bounded by $\delta^{\prime}$. It is reasonable to assume that $\delta^{\prime} \leq \delta$ since localization accuracy using GPS is usually better than using localization algorithms. 


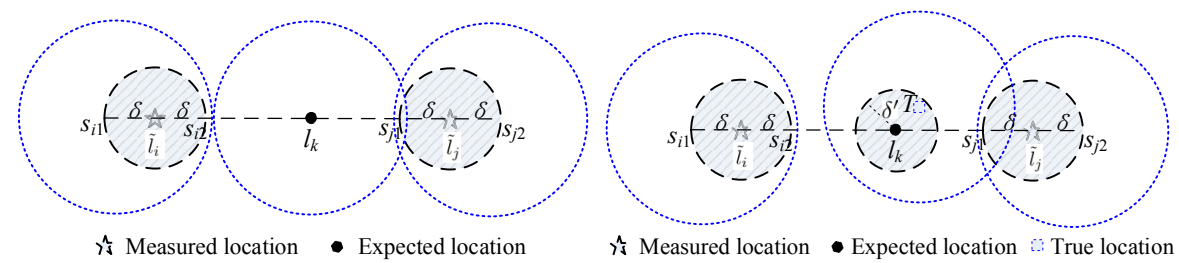

(a) When the mobile node does not have lo- (b) When the mobile node has location error cation error

Figure 5: The effects of location errors for mobile sensor nodes

\subsection{Location Error Analysis}

The barrier coverage problem is more complicated when mobile nodes also have location errors. This is because although the measured location of a mobile node shows to be the expected location, due to the location error, the true location of the node may not be the expected location. As shown in Figure 5 (a), when the mobile node $m_{k}$ does not have location error, it can move to the expected location $l_{k}$ and connect $s_{i}$ and $s_{j}$ with a guarantee. However, when the node has location error, as shown in Figure 5(b), although the measured location is $l_{k}$, the true location is actually at point $T$ (denoted by the blue square) which cannot guarantee the connection of $s_{i}$ and $s_{j}$.

Lemma 6. Given two stationary nodes $s_{i}$ and $s_{j}$, their measured locations $\tilde{l}_{i}$ and $\tilde{l}_{j}$, and $d\left(\tilde{l}_{i}, \tilde{l}_{j}\right)>2 r_{s}-2 \delta$, the minimum number of mobile nodes needed to guarantee the connection of $s_{i}$ and $s_{j}$ is $\max \left(0,\left\lceil\frac{d\left(\tilde{l}_{i}, \tilde{l}_{j}\right)-2\left(2 r_{s}-\delta-\delta^{\prime}\right)}{2 r_{s}-2 \delta^{\prime}}\right\rceil\right)+1$ when both stationary and mobile nodes have location errors.

Proof. Please see the proof in Appendix B.

Specifically, when $\delta^{\prime}=\delta$, the minimum number of mobile nodes needed to guarantee the connection of $s_{i}$ and $s_{j}$ is $\left\lceil\frac{d\left(\tilde{l}_{i}, \tilde{l}_{j}\right)}{2 s_{s}-2 \delta}\right\rceil-1$.

Recall that $N\left(s_{i}, s_{j}\right)$ is the true minimum number of mobile nodes needed to connect $s_{i}$ and $s_{j}$. Let $N_{s m}^{u}\left(s_{i}, s_{j}\right)$ and $N_{s m}^{l}\left(s_{i}, s_{j}\right)$ denote the upper and lower bound of $N\left(s_{i}, s_{j}\right)$ when both stationary and mobile nodes have location errors, respectively. Therefore, we have

$$
\begin{gathered}
N_{s m}^{u}\left(s_{i}, s_{j}\right)=\max \left(0,\left\lceil\frac{d\left(\tilde{l}_{i}, \tilde{l}_{j}\right)-2\left(2 r_{s}-\delta-\delta^{\prime}\right)}{2 r_{s}-2 \delta^{\prime}}\right\rceil\right)+1 . \\
N_{s m}^{l}\left(s_{i}, s_{j}\right)=\max \left(0,\left\lceil\frac{d\left(\tilde{l}_{i}, \tilde{l}_{j}\right)-2 \delta}{2 r_{s}}\right\rceil-1\right) .
\end{gathered}
$$

Thus, $\triangle N_{s m}\left(s_{i}, s_{j}\right)=N_{s m}^{u}\left(s_{i}, s_{j}\right)-N_{s m}^{l}\left(s_{i}, s_{j}\right)$ represents the influence on $N\left(s_{i}, s_{j}\right)$ when both stationary and mobile nodes have location errors. When $\triangle N_{s m}\left(s_{i}, s_{j}\right)=0$, the location error does not affect the minimum number of mobile nodes needed to connect $s_{i}$ and $s_{j}$. Note that $N_{s m}^{l}\left(s_{i}, s_{j}\right)=N_{s}^{l}\left(s_{i}, s_{j}\right)$ because the true location could be the expected location in the best case. 
Theorem 7. When both stationary and mobile nodes have location errors, the existence of location error does not affect the minimum number of mobile nodes needed to connect $s_{i}$ and $s_{j}$ when their measured distance $d\left(\tilde{l}_{i}, \tilde{l}_{j}\right)$ satisfies $d\left(\tilde{l}_{i}, \tilde{l}_{j}\right) \leq 2 r_{s}-2 \delta$ or $2 r_{s}+2 \delta<d\left(\tilde{l}_{i}, \tilde{l}_{j}\right)<4 r_{s}-2 \delta-2 \delta^{\prime}$ or $\left\lceil\frac{d\left(\tilde{l}_{i}, \tilde{l}_{j}\right)-2\left(2 r_{s}-\delta-\delta^{\prime}\right)}{2 r_{s}-2 \delta^{\prime}}\right\rceil+$ $1=\left\lceil\frac{d\left(\tilde{l}_{i}, \tilde{l}_{j}\right)-2 \delta}{2 r_{s}}\right\rceil-1 \geq 2$.

Proof. We omit the proof here since it is similar to the proof of Theorem 2.

Theorem 8. Considering a sensor network where both stationary and mobile nodes have location errors upper bounded by $\delta$ and $\delta^{\prime}$ respectively, at most $\left\lceil\frac{4 \delta r_{s}+\left(d-2 r_{s}-2 \delta\right) \delta^{\prime}}{2 r_{s}\left(r_{s}-\delta^{\prime}\right)}\right\rceil$ more mobile nodes are needed to connect $s_{i}$ and $s_{j}$ compared to the true minimum number of mobile nodes needed. That is, $\triangle N_{s m}\left(s_{i}, s_{j}\right) \leq$ $\left\lceil\frac{4 \delta r_{s}+\left(d-2 r_{s}-2 \delta\right) \delta^{\prime}}{2 r_{s}\left(r_{s}-\delta^{\prime}\right)}\right\rceil$ for any pair of $s_{i}$ and $s_{j}$.

Proof. The proof of this theorem is omitted since it is similar to the proof of Theorem 3.

According to Theorem 3, at most 2 more mobile nodes are needed when only stationary nodes have location errors. However, according to Theorem 8, $\triangle N_{s m}\left(s_{i}, s_{j}\right)$ is related with the measured distance, $\delta$ and $\delta^{\prime}$ when both stationary and mobile nodes have location errors. As $\delta^{\prime}$ or the measured distance increases, more mobile nodes compared to the true number of needed mobile nodes will be needed. Therefore, the existence of location error for mobile nodes could significantly influence the minimum number of mobile nodes needed to form barrier coverage.

\subsection{Progressive Mobile Node Deployment}

The progressive deployment of mobile nodes when they have location errors is similar to the progressive deployment proposed in Section 4.2.

All the expected locations of mobile nodes are set to be on the line segment of $\tilde{l}_{i}$ and $\tilde{l}_{j}$. We progressively deploy mobile nodes from the left to the right. The expected location of the first mobile node is $2 r_{s}-\left(\delta+\delta^{\prime}\right)$ away from the $\tilde{l}_{i}$. The rest of mobile nodes are progressively deployed with $2 r_{s}-2 \delta^{\prime}$ from its previously deployed mobile node. We can also easily prove that the progressive deployment is optimal which can connect $s_{i}$ and $s_{j}$ with a guarantee by using $\max \left(0,\left\lceil\frac{d\left(\tilde{l}_{i}, \tilde{l}_{j}\right)-2\left(2 r_{s}-\delta-\delta^{\prime}\right)}{2 r_{s}-2 \delta^{\prime}}\right\rceil\right)+1$ mobile nodes.

\subsection{Fault-tolerant Barrier Coverage Formation}

In order to find the minimum number of mobile nodes needed to form barrier coverage with a guarantee, we can also build a corresponding fault-tolerant barrier graph for the sensor network. Similar to the graph in Section 4.3, the left and right boundary are considered as virtual vertices $s$ and $t$, respectively. Each stationary node is modeled as a vertex. There is an edge between any pair of vertices and a weight is assigned for each edge which represents the minimum number of mobile nodes needed to connect any pair of vertices with a guarantee. 


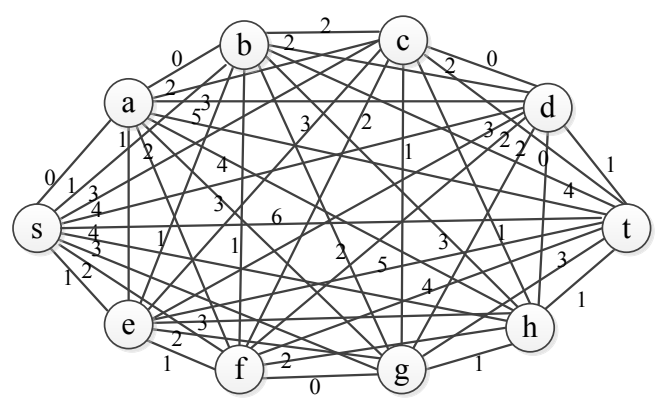

Figure 6: The FTWBG corresponding to Figure 4(a) when both stationary and mobile nodes have location errors where $r_{s}=10 \mathrm{~m}$ and $\delta=\delta^{\prime}=1 \mathrm{~m}$

Since mobile nodes also have location errors, the weight of each edge is not the same as that in Equation (2). Similar to the derivation for Equation (2), we have

$$
w\left(v_{i}, v_{j}\right)= \begin{cases}N_{s m}^{u}\left(s_{i}, s_{j}\right), & \text { if } v_{i}=s_{i} \text { and } v_{j}=s_{j}, \\ \left\lceil\frac{x_{j}-\left(r_{s}-\delta\right)}{2 r_{s}-2 \delta^{\prime}}\right\rceil, & \text { if } v_{i}=s \text { and } v_{j}=s_{j}, \\ \left\lceil\frac{L-\left(x_{j}+r_{s}-\delta\right)}{2 r_{s}-2 \delta^{\prime}}\right\rceil, & \text { if } v_{i}=t \text { and } v_{j}=s_{j}, \\ \left\lceil\frac{L}{2 r_{s}-2 \delta^{\prime}}\right\rceil, & \text { if } v_{i}=s \text { and } v_{j}=t .\end{cases}
$$

where $N_{s m}^{u}\left(s_{i}, s_{j}\right)=\max \left(0,\left\lceil\frac{d\left(\tilde{l}_{i}, \tilde{l}_{j}\right)-2\left(2 r_{s}-\delta-\delta^{\prime}\right)}{2 r_{s}-2 \delta^{\prime}}\right\rceil\right)+1$.

Similar to Theorem 4, we can prove that the minimum number of mobile nodes needed to form a barrier with a guarantee with stationary nodes when both stationary and mobile nodes have location errors is upper bounded by $\left\lceil\frac{L}{2 r_{s}-2 \delta^{\prime}}\right\rceil$.

Figure 6 shows the FTWBG when both stationary and mobile nodes have location errors. We assume that $r_{s}=10 \mathrm{~m}$ and $\delta=\delta^{\prime}=1 \mathrm{~m}$. Note that the only difference between this figure and Figure 4(b) is the weight of each edge representing the minimum number of mobile nodes needed to connect any pair of vertices with a guarantee. As shown in Figure 6, the shortest path is $s \rightarrow a \rightarrow b \rightarrow d \rightarrow t$, the length of which is $0+0+2+1=3$. Therefore, the ROI is not guaranteed to be barrier-covered after initial random deployment and 3 mobile nodes are needed to guarantee the formation of barrier coverage.

\section{Algorithm Analysis and Improvement}

In this section, we first analyze the computational complexity of the faulttolerant barrier coverage algorithm ( $\mathrm{FatBaCa})$, and then propose improving the computational efficiency of the algorithm by removing unnecessary edges on the proposed FTWBG. For the simulations in this section, we assume that only stationary nodes have location errors.

Theorem 9. The computational complexity of the FatBaCa is $O(V \ln V+E)$ where $V$ and $E$ are the sets of vertices and edges on the graph, respectively. 


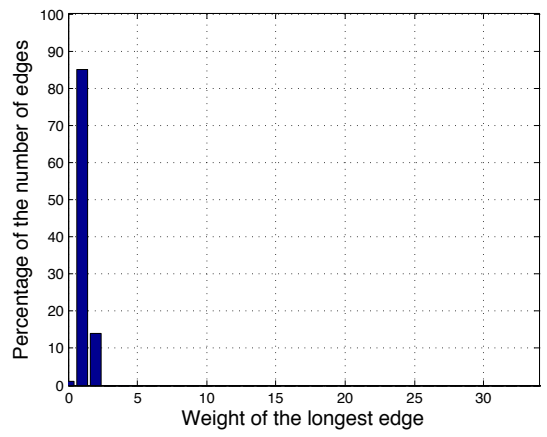

(a)

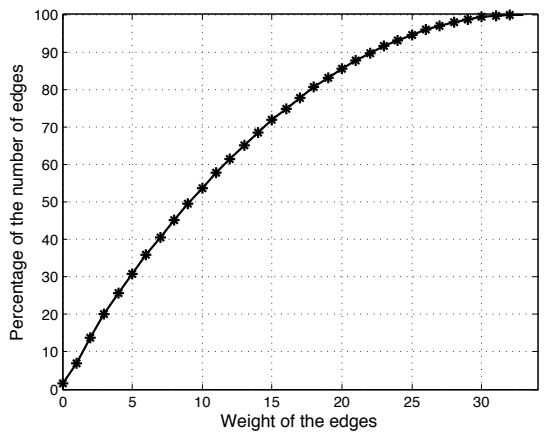

(b)

Figure 7: (a) The histogram of the longest edges of the shortest paths in 100 experiments; (b) The cumulative distribution of the weights of edges

Proof. The FatBaCa consists of two phases: constructing the FTWBG based on node deployment, and performing Dijkstra's algorithm to find the shortest path on the graph.

In order to construct the graph, we need to calculate the distance and also the minimum number of mobile nodes needed between any pair of stationary nodes. It is worth noting that the FTWBG is a complete graph. Given the graph $G=(V, E, W)$, the computational complexity of constructing the FTWBG is $O(2 E)=O(E)$, and the computational complexity of Dijkstra's algorithm is $O(V \ln V+E)$. Therefore, the computational complexity of the FatBaCa is $O(V \ln V+E)$.

Since the FTWBG is a complete graph, the computational cost becomes high when the number of deployed stationary nodes is large. However, some edges on the graph are not necessary. For example, if the distance between two stationary nodes is larger than $L$, it is not necessary to have this edge or calculate its weight.

Given a complete graph $G$, let $\operatorname{sp}(G, s, t)$ denote the shortest path from $s$ to $t$ on the graph $G$ and $l_{e}$ denote the weight of the longest edge of $s p(G, s, t)$. For a threshold $D \geq l_{e}$, if we remove all the edges longer than $D$ from the complete graph $G$ to get a new graph $G^{\prime}, s p(G, s, t)$ is also the shortest path of $G^{\prime}$. That is, all the edges longer than $l_{e}$ are not necessary for calculating the minimum number of mobile nodes needed.

To illustrate this point, Figure 7(a) shows the histogram of the longest edges of the shortest paths in 100 experiments. The ROI is a belt region of length $L=1000 \mathrm{~m}$ and width $H=100 \mathrm{~m}$. Initially, stationary nodes are randomly deployed in the ROI. We set $n=300, r_{s}=15 \mathrm{~m}$ and $\delta=3 \mathrm{~m}$. In each experiment, we find the shortest path of the graph and the longest edge on the shortest path. We can see that, although the weights of edges range from 0 to $32, l_{e} \leq 2$ for all 100 experiments, which means that there is no need to put edges with weights greater than 2 on the graph. From Figure 7(b) we can observe that only $14 \%$ edges will be needed to find the minimum cost of 
mobile nodes needed to form a barrier with a guarantee. Therefore, given 300 stationary nodes, the number of edges will be reduced from 45451 to 6363 . The computational cost will be reduced significantly by removing unnecessary edges on the graph.

Based on this observation, we argue that it is not necessary to build a complete graph in the first place, and the computational efficiency of the FatBaCa can be improved by removing unnecessary edges of the graph. Although $l_{e}$ is unknown to us without the complete graph, we can find a suitable value for $D$ to remove as many unnecessary edges as possible. The closer $D$ and $l_{e}$ are, the better is the removal of unnecessary edges. Note that $D$ is a weight threshold for edge removal. Let $D T$ denote the distance threshold corresponding to $D$. That is, the weight threshold $D$ can be calculated from the distance threshold $D T$ according to Equation (5).

The improved algorithm is described as follows.

- Step 1: Calculate the measured distance between any pair of nodes.

- Step 2: If the measured distance is no larger than $D T$, add the corresponding edge on the graph and calculate its weight according to Equation (5); otherwise, do not add the edge on the graph.

- Step 3: Check whether the graph has a path from $s$ to $t$. If there is a path, find the shortest path; otherwise, increase $D T$ by $2 r_{s}$ and go to step 2 .

The improved algorithm reduces the computational cost from two aspects. First, instead of calculating the weight for all edges, we only need to calculate the weights for edges no larger than $D T$. Second, the computational cost for finding the shortest path of the graph is reduced since the number of edges has been decreased.

As mentioned earlier, $D=l_{e}$ is the optimal case and we can calculate the optimal value for $D T$ accordingly. However, $l_{e}$ is unknown to us since we would not construct the complete graph in the improved algorithm. Since nodes are randomly deployed in the network, it is difficult to analyze the mathematical relationship between $l_{e}$ and the node deployment. However, generally speaking, $l_{e}$ is related to the node density and the sensing range, which can be seen from Figures 8 and 9. The higher node density, the smaller the optimal value for $D T$; the larger sensing range, the smaller the optimal value for $D T$.

Figures 8 and 9 show the optimal value for $D T$ versus the number of stationary nodes and the sensing range of nodes, respectively. The default values are $r_{s}=15 \mathrm{~m}, \delta=3 \mathrm{~m}$, and $n=300.100$ experiments are conducted and each point on the figure is an average of 100 experiments. As shown in Figure 8 , the optimal value for $D T$ decreases when $n$ increases since nodes are closer to each other when more nodes are deployed. Meanwhile, the ratio of edges in the new graph compared to the original complete graph is also decreasing as $n$ increases, and only $8 \%$ of edges of the original graph will be left in the new graph when 400 stationary nodes are deployed in the sensor network, which significantly reduces the computational cost. We obtain similar observations as 


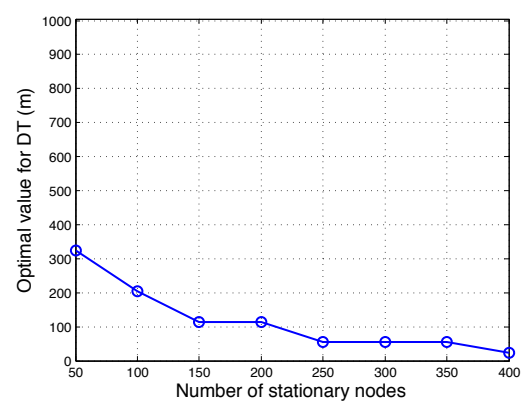

(a)

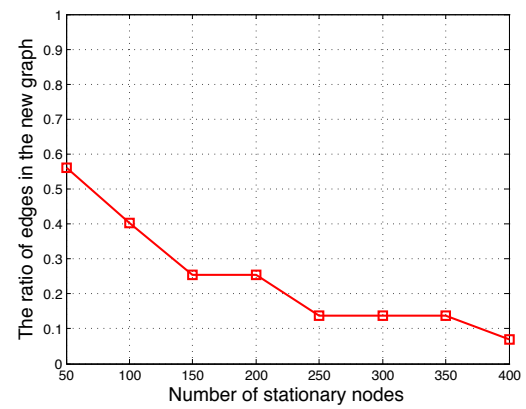

(b)

Figure 8: (a) The optimal value for $D T$; (b) The ratio of the number of edges in the new graph to the number of edges in the original complete graph; when the number of stationary nodes changes

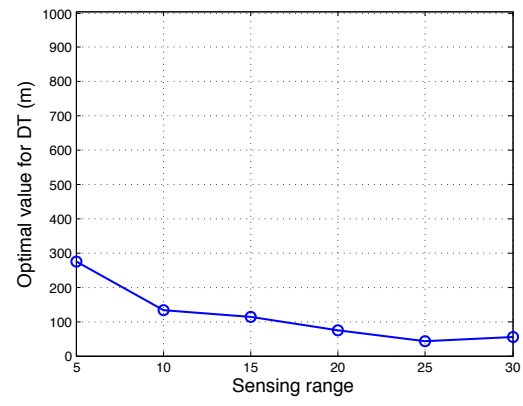

(a)

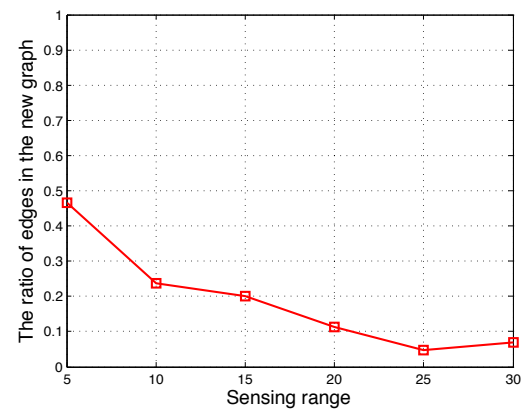

(b)

Figure 9: (a) The optimal value for DT; (b) The ratio of the number of edges in the new graph to the number of edges in the original complete graph; when the sensing range changes

in Figure 8. Both the optimal value for $D T$ and the ratio of edges in the new graph decrease when the sensing range increases, which is because nodes are closer to each other when their sensing ranges are larger.

Figure 10 shows the performance results when we change the value of $D T$ in the improved algorithm. We set $n=300, r_{s}=15 \mathrm{~m}$ and $\delta=3 \mathrm{~m}$. DT varies from $50 \mathrm{~m}$ to $1000 \mathrm{~m}$ with step size of $50 \mathrm{~m} .100$ experiments are conducted and each point on the figure is an average of 100 experiments.

It is worth noting that, when $D T \leq 100 \mathrm{~m}$, more mobile nodes will be needed for the improved algorithm compared to the original algorithm. This is because the shortest path on the improved graph is not the shortest path on the original graph when too many edges are removed. Meanwhile, more than $80 \%$ edges on the original graph will be removed when $D T \leq 100 \mathrm{~m}$, which can significantly reduce the computational cost.

We can also observe that, when $D T \geq 150 \mathrm{~m}$, the improved algorithm not only improves the computational efficiency, but also requires the same number 


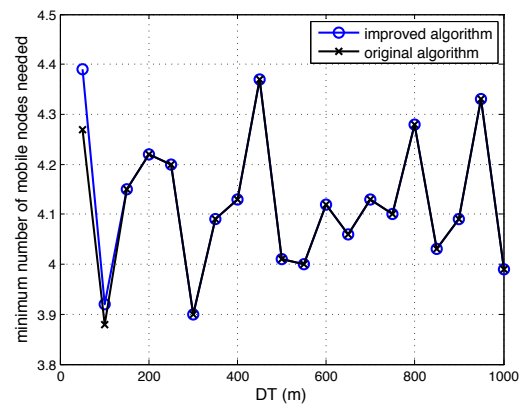

(a)

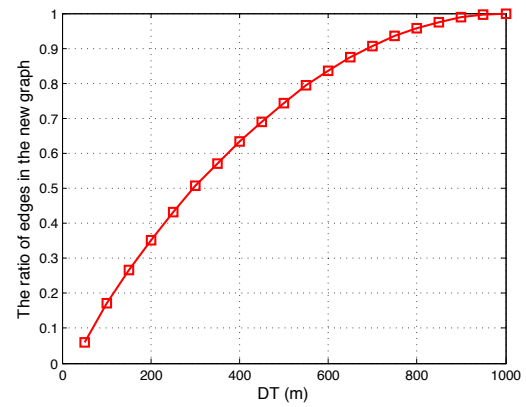

(b)

Figure 10: (a) The required minimum number of mobile nodes; (b) The ratio of the number of edges in the new graph to the number of edges in the original complete graph; when $D T$ changes

of mobile nodes with the original algorithm. Therefore, by choosing a suitable $D T$, the optimized algorithm can find the minimum required number of mobile nodes needed to form a barrier with a guarantee while simultaneously reducing the computational cost.

\section{Performance Evaluation}

In this section, we conduct extensive simulations to evaluate the effects of location errors on barrier coverage and the performance of the proposed Fat$\mathrm{BaCa}$ using Matlab. Note that, we use the proposed original algorithm instead of the improved algorithm in the simulations in order to evaluate the effects of different parameters. The ROI is a belt region of length $L=1000 \mathrm{~m}$ and width $H=100 \mathrm{~m}$. Initially, stationary nodes are randomly deployed in the ROI. After the minimum number of mobile nodes is calculated, mobile nodes are deployed and scheduled to form barrier coverage with predeployed stationary nodes. The performance evaluation mainly focuses on the following metrics:

- The probability of barrier coverage which is represented by the ratio of the number of times that the ROI is already barrier-covered for initial deployment of sensor nodes to the total number of experiments;

- The influence of location errors on the minimum number of mobile nodes needed to connect any pair of stationary nodes;

- The minimum number of mobile nodes needed to form barrier coverage;

- The total cost needed to form barrier coverage, including the cost of both stationary and mobile nodes.

We evaluate the number of stationary nodes $n$, the sensing range $r_{s}, \delta$ and $\delta^{\prime}$ for these metrics. In our simulations, we set $n=300, r_{s}=15 \mathrm{~m}, \delta=3$ $\mathrm{m}, \delta^{\prime}=1 \mathrm{~m}$ and $D T=L / 5$ in default. When we evaluate the effects of one 


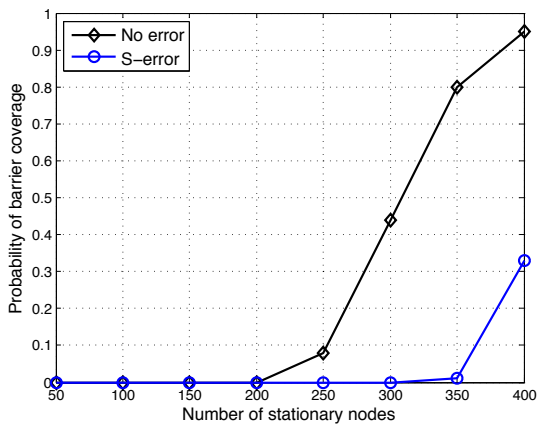

(a) $r_{s}=15 \mathrm{~m}, \delta=3 \mathrm{~m}$ and $\delta^{\prime}=1 \mathrm{~m}$

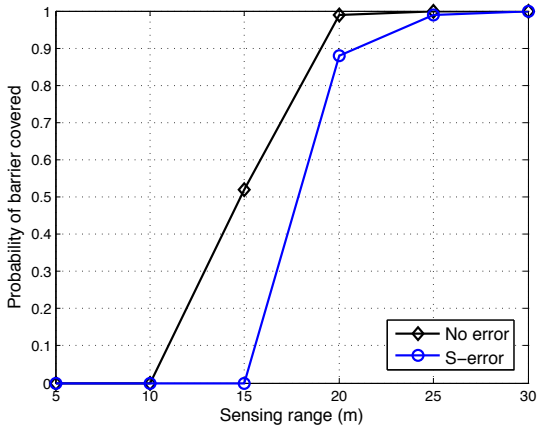

(b) $n=300, \delta=3 \mathrm{~m}$ and $\delta^{\prime}=1 \mathrm{~m}$

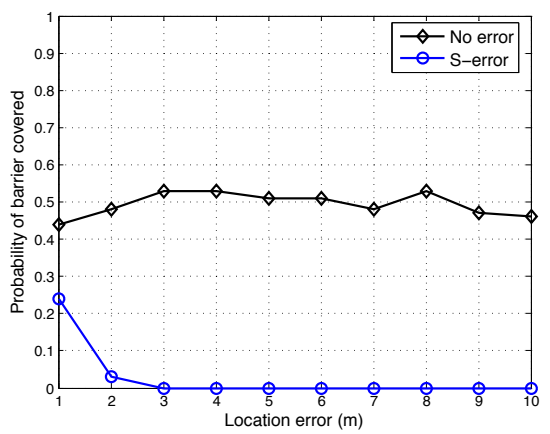

(c) $n=300$ and $r_{s}=15 \mathrm{~m}$ and $\delta^{\prime}=1 \mathrm{~m}$

Figure 11: The probability of barrier coverage after initial node deployment. "No error" means that nodes do not have location errors, "S-error" means that only stationary nodes have location errors.

parameter, the other parameters do not change. Three scenarios are evaluated in our simulations: when no node has location error, when only stationary nodes have location errors, and when both stationary and mobile nodes have location errors. For all the simulation results in Figures 11, 13 and 14, each data point is an average of 100 experiments. For all the simulation results in Figure 12, each data point is the maximum value of 100 experiments.

\subsection{Probability of Barrier Coverage}

Figure 11 shows the effects of different parameters on the probability of barrier coverage when the network has only stationary nodes. As shown in Figures 11(a) and 11(b), the probability of barrier coverage increases as the number of stationary nodes or the sensing range increases. As shown in Figure 11(c), the probability of barrier coverage decreases as the location error increases. Moreover, the probability of barrier coverage is much lower when the nodes have location errors than when the nodes do not have location errors. Therefore, it is necessary to deploy mobile nodes to form a barrier with a guarantee. 


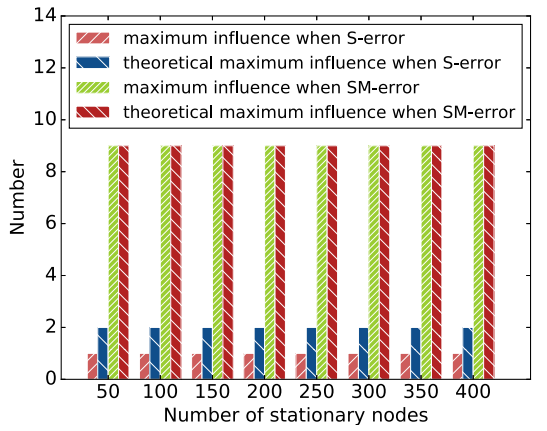

(a) $r_{s}=15 \mathrm{~m}, \delta=3 \mathrm{~m}$ and $\delta^{\prime}=1 \mathrm{~m}$

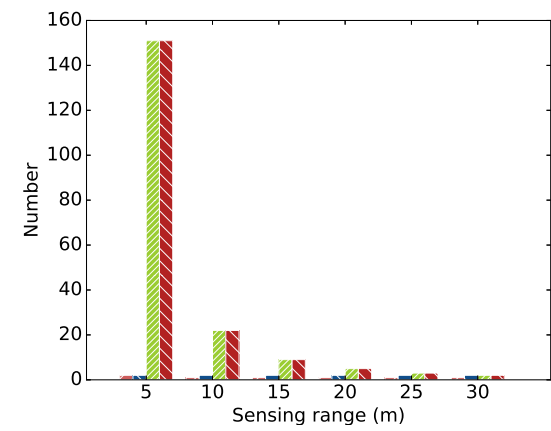

(b) $n=300, \delta=3 \mathrm{~m}$ and $\delta^{\prime}=1 \mathrm{~m}$

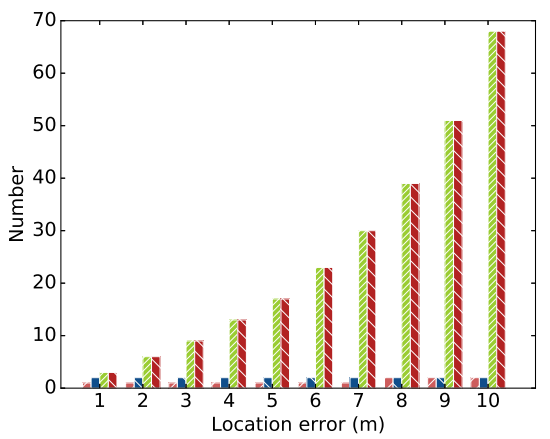

(c) $n=300, r_{s}=15 \mathrm{~m}$ and $\delta^{\prime}=1 \mathrm{~m}$

Figure 12: The influence of location errors on the number of mobile nodes needed to connect two stationary nodes. "SM-error" means that both stationary and mobile nodes have location error

7.2. The Influence of Location Errors on The Number of Mobile Nodes Needed to Connect Two Stationary Nodes

$\triangle N_{s}\left(s_{i}, s_{j}\right)$ and $\triangle N_{s m}\left(s_{i}, s_{j}\right)$ represent the influence of location errors on the number of mobile nodes needed to connect two stationary nodes $s_{i}$ and $s_{j}$ when only stationary nodes have location errors and when both stationary and mobile nodes have location errors, respectively. Figure 12 shows the effects of different parameters on $\triangle N_{s}\left(s_{i}, s_{j}\right)$ and $\triangle N_{s m}\left(s_{i}, s_{j}\right)$ and also their theoretical upper bound. First we can observe that the maximum of $\triangle N_{s}\left(s_{i}, s_{j}\right)$ when only stationary nodes have location errors is always no larger than 2 , which validates the correctness of Theorem 3 . We then observe that the maximum of $\triangle N_{s m}\left(s_{i}, s_{j}\right)$ when both stationary and mobile nodes have location errors is always no larger than its theoretical upper bound, which validates the correctness of Theorem 8.

As shown in Figure 12(a), the maximum of $\triangle N_{s m}\left(s_{i}, s_{j}\right)$ does not change when the number of stationary nodes increases. This is because the largest distance of two stationary nodes is almost always the length of the area. Figure 12(b) shows that the maximum of $\triangle N_{s m}\left(s_{i}, s_{j}\right)$ decreases when the sensing range increases, which implies that the influence of location error is smaller 


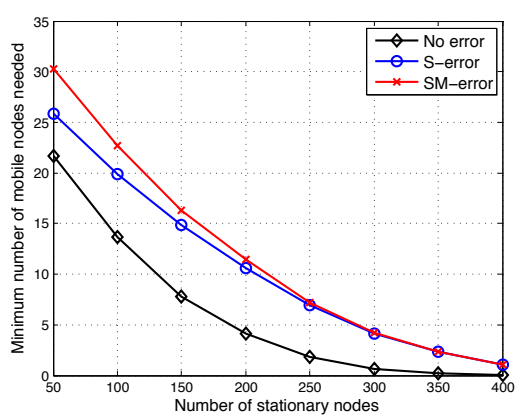

(a) $r_{s}=15 \mathrm{~m}, \delta=3 \mathrm{~m}$ and $\delta^{\prime}=1 \mathrm{~m}$

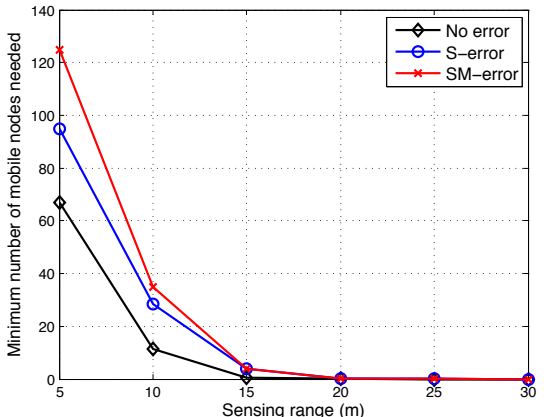

(b) $n=300, \delta=3 \mathrm{~m}$ and $\delta^{\prime}=1 \mathrm{~m}$

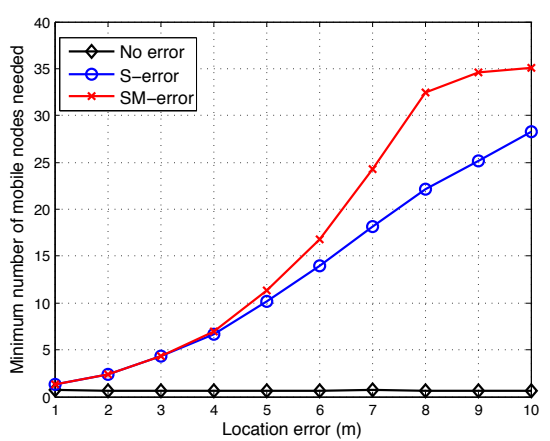

(c) $n=300, r_{s}=15 \mathrm{~m}$ and $\delta^{\prime}=1 \mathrm{~m}$

Figure 13: The effects of different parameters on the minimum number of mobile nodes needed to form a barrier.

for larger sensing range. Figure 12(c) shows that the maximum $\triangle N_{s m}\left(s_{i}, s_{j}\right)$ increases as the location error increases, which implies that the influence of location error becomes more serious when the location error becomes larger.

\subsection{Minimum Number of Mobile Nodes Needed}

Figure 13 shows the effects of different parameters on the minimum number of mobile nodes needed to form barrier coverage with a guarantee. As shown in Figures 13(a) and 13(b), we can see that the minimum number of mobile nodes needed decreases as the number or the sensing range of nodes increases. This is because a larger number of stationary nodes deployed or a larger sensing range can reduce the number of gaps between stationary nodes as well as the sizes of gaps. We can observe that when there are many deployed stationary nodes or the sensing range is large enough, the sensor network can provide barrier coverage even when nodes have location errors.

From Figure 13(c), we can observe that the minimum number of mobile nodes needed increases when the location error increases. This is because larger location error results in larger uncertainty of a location and therefore requires more mobile nodes. Moreover, when $\delta$ increases, the required number of mobile nodes when both stationary and mobile nodes have location errors increases 


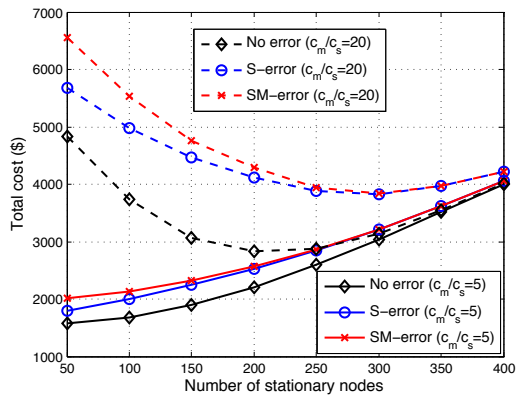

(a) $r_{s}=15 \mathrm{~m}, \delta=3 \mathrm{~m}$ and $\delta^{\prime}=1 \mathrm{~m}$

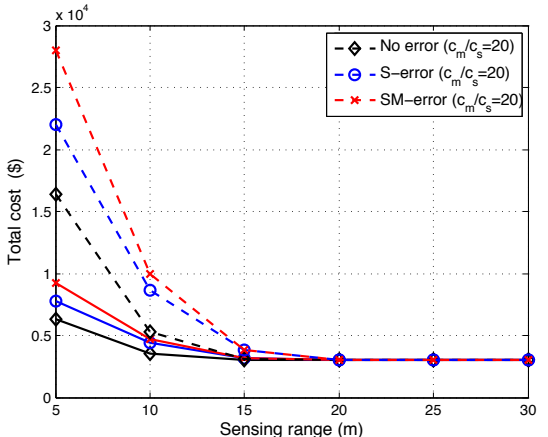

(b) $n=300, \delta=3 \mathrm{~m}$ and $\delta^{\prime}=1 \mathrm{~m}$

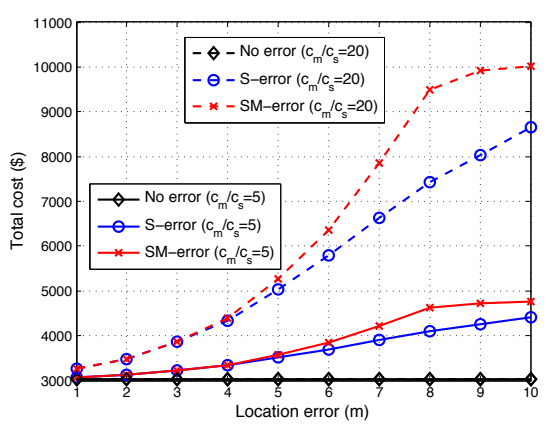

(c) $n=300, r_{s}=15 \mathrm{~m}$ and $\delta^{\prime}=1 \mathrm{~m}$

Figure 14: The effects of different parameters on the total cost to form a barrier

faster than that when only stationary nodes have location errors, which implies that the influence of location errors is more serious when both stationary and mobile nodes have location errors. We can also observe that the required number of mobile nodes when both stationary and mobile nodes have location errors is usually larger than when only stationary node have location errors.

\subsection{Total Cost Needed}

The total cost needed to form a barrier is the sum of the cost of deployed stationary nodes and the cost of mobile nodes needed. Let $c_{s}$ and $c_{m}$ denote the cost of a stationary node and a mobile node, respectively. For simplicity, we assume $c_{s}=\$ 10$ for a stationary node.

As shown in Figure 14(a), when mobile nodes are not very expensive (e.g., $\left.c_{m} / c_{s}=5\right)$, the total cost mainly depends on the number of deployed stationary nodes. Therefore, the total cost increases as the number of deployed stationary nodes increase when $c_{m} / c_{s}=5$. However, when mobile nodes are much more expensive than stationary nodes (e.g., $c_{m} / c_{s}=20$ ), the number of mobile nodes needed can significantly affect the total cost needed. For example, the total cost for $n=50$ is much larger than that for $n=200$ because the former needs much more mobile nodes to form a barrier. For the simulated belt region, the total 
cost reaches the minimum when 200 stationary nodes are deployed. We can conclude that, given an ROI, the number of stationary nodes to be deployed highly depends on $c_{m} / c_{s}$.

We can see from Figure 14(b) that the total cost needed decreases when the sensing range of nodes increases, which is because the number of mobile nodes needed decreases. As shown in Figure 14(c), the total cost needed increases when the location error increases, which is because more mobile nodes are needed for a larger location error.

\section{Conclusions and Future Work}

In this paper, we made the first attempt to explore the effects of location errors on barrier coverage and leveraged mobile nodes to fill in gaps between stationary nodes to form a barrier with a guarantee when nodes have location errors. When only stationary nodes have location errors, at most 2 more mobile nodes are needed compared to the true minimum number of nodes needed to connect any pair of stationary nodes with a guarantee. However, when both stationary and mobile nodes have location errors, the difference between the minimum number of mobile nodes needed and the true minimum number of mobile nodes needed is related to length of the belt region and the location error. We proposed a progressive method that uses exactly the same minimum number of mobile nodes derived in theory to connect any pair of nodes with a guarantee.

Furthermore, we proposed a fault-tolerant weighted barrier graph to model the barrier coverage formation problem when nodes have location errors, and proved that the minimum number of mobile nodes needed to form barrier coverage with a guarantee is the length of the shortest path on the graph. A faulttolerant barrier coverage algorithm was proposed to realize barrier coverage formation. In order to further reduce the computational cost of the algorithm, we optimize the algorithm by removing unnecessary edges from the complete graph. Extensive simulation results validated the correctness of our analysis and the proposed algorithms.

This paper used a rather simple but practical location error model, which provides researchers interested in this work with a very good starting point to continue exploiting more practical scenarios and complicated location error models for barrier coverage.

In reality, sensor nodes may be deployed in harsh environments, such as hills and lakes. In these scenarios, we cannot deploy mobile nodes in a straight line to fill in gaps. Instead, a curve-based mobile node deployment should be studied. The effects of location errors based on line-based deployment may not hold for curve-based deployment. However, the analysis idea and the proposed FTWBG model can be further used for curve-based barrier coverage when nodes have location errors. He et al. [12] have studied the barrier coverage of curve-based deployment instead of line-based deployment. Thus, based on the work in [12] and the work in this paper, we would like to study the curve-based barrier coverage formation problem when nodes have location errors in the future. 
Another issue is that the location errors may follow some distribution, e.g., the Gaussian. Instead of forming a barrier with a guarantee, one may investigate how many mobile nodes are needed to achieve barrier coverage at some confidence level given that location errors follow some distribution. This problem is very interesting but also very complicated. The analysis results in this paper would not hold if location errors follow some distribution, and we think that more work should be conducted in this direction.

\section{Acknowledgements}

The authors would like to thank the referees whose insightful comments have helped improve the presentation of this paper significantly. This work was supported in part by National Natural Science of China (Grants No. 61502352, 61309023 and 61373167), National Basic Research Program of China (Grant No. 2014CB340600), US National Science Foundation (Grant No. 0953238), Natural Science Foundation of Hubei Province and Jiangsu Province (Grants No. 2015CFB203 and BK20150383), Shandong Provincial Key Program of Research and Development (Grant No.2015GGX101045), Qingdao Fundamental Research

Project (Grant No.15-9-1-79-jch), and the Fundamental Research Funds for the Central Universities (Grants No. 2042015kf0016 and 2042016kf0190).

\section{References}

[1] S. Kumar, T. H. Lai, A. Arora, Barrier Coverage with Wireless Sensors, in: Proc. of ACM MobiCom, 2005, pp. 284-298.

[2] K. Dantu, M. H. Rahimi, H. Shah, S. Babel, A. Dhariwal, G. S. Sukhatme, Robomote: Enabling Mobility in Sensor Networks, in: Proc. of IEEE IPSN, 2005, pp. 404-409.

[3] A. A. Somasundara, A. Ramamoorthy, Mobile Element Scheduling with Dynamic Deadlines, IEEE Transactions on Mobile Computing 6 (4) (2007) $1142-1157$.

[4] B. Hofmann-Wellenhof, H. Lichtenegger, J. Collins, Global Positioning System, Theory and Practice 1.

[5] H. Pirzadeh, Computational Geometry with the Rotating Calipers, Master Thesis.

[6] P. Bahl, V. N. Padmanabhan, RADAR: An In-Building RF-based User Location and Tracking System, in: Proc. of IEEE INFOCOM, Vol. 2, 2000, pp. $775-784$.

[7] D. Niculescu, B. Nath, DV Based Positioning in Ad Hoc Networks, Telecommunication Systems 22 (1-4) (2003) 267-280. 
[8] H. Chen, W. Lou, Z. Wang, J. Wu, Z. Wang, A. Xia, Securing dv-hop localization against wormhole attacks in wireless sensor networks, Pervasive and Mobile Computing 16 (2015) 22-35.

[9] T. He, C. Huang, B. M. Blum, J. A. Stankovic, T. Abdelzaher, Range-free Localization Schemes for Large Scale Sensor Networks, in: Proc. of ACM MobiCom, 2003, pp. 81-95.

[10] J. Shen, Z. Wang, Z. Wang, Fault tolerant line-based barrier coverage formation in mobile wireless sensor networks, International Journal of Distributed Sensor Networks 2015. doi:10.1155/2015/930585.

[11] B. Liu, O. Dousse, J. Wang, A. Saipulla, Strong Barrier Coverage of Wireless Sensor Networks, in: Proc. of ACM MobiHoc, 2008, pp. 411-420.

[12] S. He, X. Gong, J. Zhang, J. Chen, Y. Sun, Curve-based deployment for barrier coverage in wireless sensor networks, IEEE Transactions on Wireless Communications 13 (2) (2014) 724-735.

[13] B. Wang, H. Xu, W. Liu, H. Liang, A novel node placement for long belt coverage in wireless networks, IEEE Transactions on Computers 62 (12) (2013) 2341-2353.

[14] B. Wang, H. Xu, W. Liu, L. T. Yang, The optimal node placement for long belt coverage in wireless networks, IEEE Transactions on Computers 64 (2) (2015) 587-592.

[15] J. Chen, B. Wang, W. Liu, Constructing perimeter barrier coverage with bistatic radar sensors, Journal of Network and Computer Applications 57 (2015) 129-141.

[16] H. Kim, J. Son, H. J. Chang, H. Oh, Event-driven partial barriers in wireless sensor networks, in: Proc. of IEEE ICNC, 2016, pp. 1-5.

[17] H. Kim, J. Ben-Othman, On resilient event-driven partial barriers in mobile sensor networks, in: Proc. of IEEE ICC, 2016, pp. 1-6.

[18] B. Wang, Coverage problems in sensor networks: A survey, ACM Computing Surveys 43 (4) (2011) 32.

[19] F. Wu, Y. Gui, Z. Wang, X. Gao, G. Chen, A survey on barrier coverage with sensors, Frontiers of Computer Science 2016. doi:10.1007/s11704-016$5532-4$.

[20] C. Shen, W. Cheng, X. Liao, S. Peng, Barrier Coverage with Mobile Sensors, in: Proc. of I-SPAN, no. 2006, 2008, pp. 99-104.

[21] Y. Keung, B. Li, Q. Zhang, The Intrusion Detection in Mobile Sensor Network, in: Proc. of ACM MobiHoc, 2010, pp. 11-20. 
[22] L. Kong, X. Liu, Z. Li, M.-Y. Wu, Automatic barrier coverage formation with mobile sensor networks, in: Proc. of IEEE ICC, 2010, pp. 1-5.

[23] D. Z. Chen, Y. Gu, J. Li, H. Wang, Algorithms on minimizing the maximum sensor movement for barrier coverage of a linear domain, Discrete Computational Geometry (50) (2013) 374-408.

[24] S. Silvestri, K. Goss, Mobibar: an autonomous deployment algorithm for barrier coverage with mobile sensors, Ad Hoc Networks.

[25] K. Liu, Y. Zhuang, Z. Wang, J. Ma, Spatiotemporal correlation based faulttolerant event detection in wireless sensor networks, International Journal of Distributed Sensor Networks 2015. doi:10.1155/2015/643570.

[26] S. He, J. Chen, X. Li, X. S. Shen, Y. Sun, Mobility and intruder prior information improving the barrier coverage of sparse sensor networks, IEEE Transactions on Mobile Computing 13 (6) (2014) 1268-1282.

[27] S. He, D.-H. Shin, J. Zhang, J. Chen, Y. Sun, Full-view area coverage in camera sensor networks: Dimension reduction and near-optimal solutions, IEEE Transactions on Vehicular Technology 65 (9) (2016) 7448 - 7461.

[28] Q. Yang, S. He, J. Li, J. Chen, Y. Sun, Energy-efficient probabilistic area coverage in wireless sensor networks, IEEE Transactions on Vehicular Technology 64 (1) (2015) 367-377.

[29] J. Tian, X. Liang, G. Wang, Deployment and Reallocation in Mobile Survivability-Heterogeneous Wireless Sensor Networks for Barrier Coverage, Ad Hoc Networks 36 (2016) 321-331.

[30] A. Saipulla, B. Liu, J. Wang, Finding and mending barrier gaps in wireless sensor networks, in: Proc. of IEEE Globecom, 2010, pp. 1-5.

[31] A. Saipulla, B. Liu, G. Xing, X. Fu, J. Wang, Barrier Coverage with Sensors of Limited Mobility, in: Proc. of ACM MobiHoc, 2010, pp. 201-210.

[32] Z. Wang, J. Liao, Q. Cao, H. Qi, Z. Wang, Achieving k-barrier Coverage in Hybrid Directional Sensor Networks, IEEE Transactions on Mobile Computing 13 (7) (2014) 1443-1455.

[33] Y. Zhang, X. Sun, B. Wang, Efficient algorithm for k-barrier coverage based on integer linear programming, China Communications 13 (7) (2016) 1623 .

[34] Z. Wang, H. Chen, Q. Cao, H. Qi, Z. Wang, Fault Tolerant Barrier Coverage for Wireless Sensor Networks, in: Proc. of IEEE INFOCOM, 2014, pp. 1869-1877.

[35] T. H. Cormen, C. E. Leiserson, R. L. Rivest, Clifford Stein, Introduction to Algorithms, 2009. 
[36] E. L. Lawler, Combinatorial Optimization: Networks and Matroids, Holt, Rinehart and Winston, New York, 1976.

\section{Appendix A. Proof of Theorem 3}

Proof. $\triangle N_{s}\left(s_{i}, s_{j}\right)$ represents the influence of location error on the minimum number of mobile sensor nodes needed. We prove the theorem from the following cases.

Case 1: When $d\left(\tilde{l}_{i}, \tilde{l}_{j}\right)+2 \delta \leq 2 r_{s}, \triangle N_{s}\left(s_{i}, s_{j}\right)=0$.

Case 2: When $d\left(\tilde{l}_{i}, \tilde{l}_{j}\right)+2 \delta>2 r_{s}$ and $d\left(\tilde{l}_{i}, \tilde{l}_{j}\right)-2 \delta \leq 2 r_{s}, N_{s}^{u}\left(s_{i}, s_{j}\right)=$ $\left\lceil\frac{d\left(\tilde{l}_{i}, \tilde{l}_{j}\right)+2 \delta}{2 r_{s}}\right\rceil-1$ and $N_{s}^{l}\left(s_{i}, s_{j}\right)=0$. Therefore $\triangle N_{s}\left(s_{i}, s_{j}\right)=\left\lceil\frac{d\left(\tilde{l}_{i}, \tilde{l}_{j}\right)+2 \delta}{2 r_{s}}\right\rceil-1$. Since $d\left(\tilde{l}_{i}, \tilde{l}_{j}\right)+2 \delta>2 r_{s},\left\lceil\frac{d\left(\tilde{l}_{i}, \tilde{l}_{j}\right)+2 \delta}{2 r_{s}}\right\rceil \geq 2$. Since $d\left(\tilde{l}_{i}, \tilde{l}_{j}\right)-2 \delta \leq 2 r_{s}, d\left(\tilde{l}_{i}, \tilde{l}_{j}\right)+$ $2 \delta \leq 2 r_{s}+4 \delta<6 r_{s}$ and then $\left\lceil\frac{d\left(\tilde{l}_{i}, \tilde{l}_{j}\right)+2 \delta}{2 r_{s}}\right\rceil \leq 3$. Therefore, $1 \leq \triangle N_{s}\left(s_{i}, s_{j}\right) \leq 2$.

Case 3 : When $d\left(\tilde{l}_{i}, \tilde{l}_{j}\right)+2 \delta>2 r_{s}$ and $d\left(\tilde{l}_{i}, \tilde{l}_{j}\right)-2 \delta>2 r_{s}$, we have

$$
\begin{aligned}
\triangle N_{s}\left(s_{i}, s_{j}\right) & =\left\lceil\frac{d\left(\tilde{l}_{i}, \tilde{l}_{j}\right)+2 \delta}{2 r_{s}}\right\rceil-\left\lceil\frac{d\left(\tilde{l}_{i}, \tilde{l}_{j}\right)-2 \delta}{2 r_{s}}\right\rceil \\
& \leq\left\lceil\frac{d\left(\tilde{l}_{i}, \tilde{l}_{j}\right)+2 r_{s}}{2 r_{s}}\right\rceil-\left\lceil\frac{d\left(\tilde{l}_{i}, \tilde{l}_{j}\right)-2 r_{s}}{2 r_{s}}\right\rceil=2
\end{aligned}
$$

In all cases, $\triangle N_{s}\left(s_{i}, s_{j}\right) \leq 2$ which means that at most 2 more mobile nodes are needed compared to $N\left(s_{i}, s_{j}\right)$ when only stationary nodes have location errors.

\section{Appendix B. Proof of Lemma 6}

Proof. In order to use as few mobile nodes as possible, the expected locations of mobile nodes should be on the line segment $\tilde{l}_{i} \tilde{l}_{j}$. Note that an expected location in this case is a location where a mobile node should move to, which is indeed a measured location. According to Lemma 1, two mobile nodes overlap with each other with a guarantee only if their measured distance is no larger than $2 r_{s}-2 \delta^{\prime}$. A stationary node and a mobile node overlap with each with a guarantee only if their measured distance is no larger than $2 r_{s}-\left(\delta+\delta^{\prime}\right)$. We consider the following two cases.

Case 1: when only 1 mobile node is needed, the measured distance between the mobile node and $s_{i}$ or $s_{j}$ should be no larger than $2 r_{s}-\delta-\delta^{\prime}$. Therefore, $d\left(\tilde{l}_{i}, \tilde{l}_{j}\right)$ should satisfy that $2 r_{s}-2 \delta<d\left(\tilde{l}_{i}, \tilde{l}_{j}\right) \leq 2\left(2 r_{s}-\delta-\delta^{\prime}\right)$.

Case 2 : when $d\left(\tilde{l}_{i}, \tilde{l}_{j}\right)>2\left(2 r_{s}-\delta-\delta^{\prime}\right)$, more than one mobile nodes are needed, and the measured distance between any two mobile nodes should be less than $2 r_{s}-2 \delta^{\prime}$. Therefore, the minimum number of mobile nodes needed to guarantee the connection of $s_{i}$ and $s_{j}$ is $\left\lceil\frac{d\left(\tilde{l}_{i}, \tilde{l}_{j}\right)-2\left(2 r_{s}-\delta-\delta^{\prime}\right)}{2 r_{s}-2 \delta^{\prime}}\right\rceil+1$.

By combining the two cases together, the minimum number of mobile nodes needed to guarantee the connection of $s_{i}$ and $s_{j}$ is $\max \left(0,\left\lceil\frac{d\left(\tilde{l}_{i}, \tilde{l}_{j}\right)-2\left(2 r_{s}-\delta-\delta^{\prime}\right)}{2 r_{s}-2 \delta^{\prime}}\right\rceil\right)+$ 1 when $d\left(\tilde{l}_{i}, \tilde{l}_{j}\right)>2 r_{s}-2 \delta$. 


\section{Vita}

Zhibo Wang received the B.E. degree in Automation from Zhejiang University, China, in 2007, and his Ph.D degree in Electrical Engineering and Computer Science from University of Tennessee, Knoxville, in 2014. He is currently an Associate Professor with the School of Computer, Wuhan University, China. His currently research interests include wireless sensor networks and mobile sensing systems. He is a member of IEEE and ACM.

Honglong Chen received his BE degree in automation from China University of Petroleum, China, in 2006, his ME degree in the Department of Control from Zhejiang University, China, in 2008, and his PhD degree in computer science from The Hong Kong Polytechnic University, Hong Kong, in 2012. He is currently an associate professor in the College of Information and Control, China University of Petroleum, China. His research interests are in the areas of wireless sensor networks, delay tolerant networks, security and privacy.

Qing Cao received his Ph.D. degree from the University of Illinois in 2008, his M.S. degree from the University of Virginia, and his B.S. degree from Fudan University, China. He is currently an Assistant Professor in the Department of Electrical Engineering and Computer Science at the University of Tennessee, Knoxville. His research interests include wireless sensor networks, embedded systems, and distributed networks. He is a member of ACM and IEEE.

Hairong Qi received the B.S. and M.S. degrees in Computer Science from Northern JiaoTong University, Beijing, China in 1992 and 1995 respectively, and the Ph.D. degree in Computer Engineering from North Carolina State University, Raleigh, in 1999. She is currently the Gonzalez Family Professor with the Department of Electrical Engineering and Computer Science at the University of Tennessee, Knoxville. Her current research interests are in advanced imaging and collaborative processing in resource-constrained distributed environment, hyperspectral image analysis, and bioinformatics. She is a senior member of IEEE and a member of a member of Sigma Xi.

Zhi Wang received the B.S. degree from Shenyang Jian Zhu University, China, in 1991, the M.S. degree from Southeast University, China, in 1997, and the Ph.D. degree from Shenyang Institute of Automation, the Chinese Academy of Sciences, China, in 2000. He is currently an Associate Professor in the State Key Laboratory of Industrial Control Technology, Zhejiang University. His research interests include wireless sensor networks, and industrial communication and systems. He is a member of IEEE.

Qian Wang received the B.S. degree from Wuhan University, China, in 2003, the M.S. degree from Shanghai Institute of Microsystem and Information Technology, Chinese Academy of Sciences, China, in 2006, and the Ph.D. degree from Illinois Institute of Technology, USA, in 2012, all in Electrical Engineering. He is currently a Professor with the School of Computer Science, Wuhan University. His research interests include wireless network security and privacy, cloud computing security, and applied cryptography. Qian is an expert under "1000 Young Talents Program" of China. He is a co-recipient of the Best Paper Award from IEEE ICNP 2011. He is a Member of the IEEE and a Member of the ACM. 


\section{Photo}

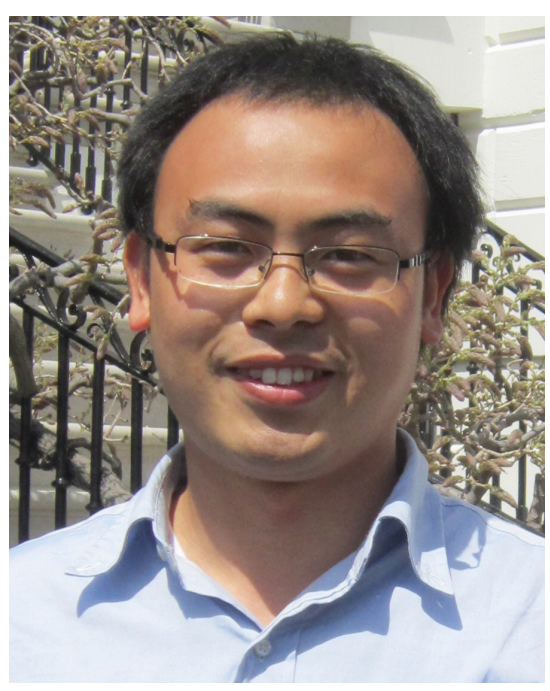

(a) Zhibo Wang

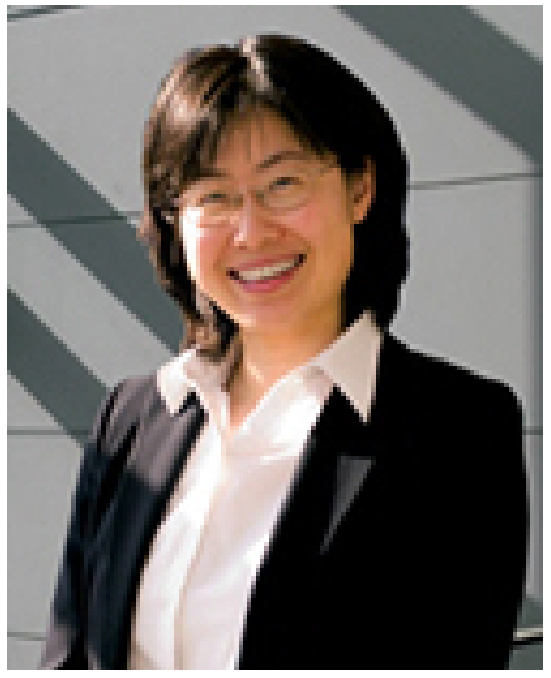

(d) Hairong Qi

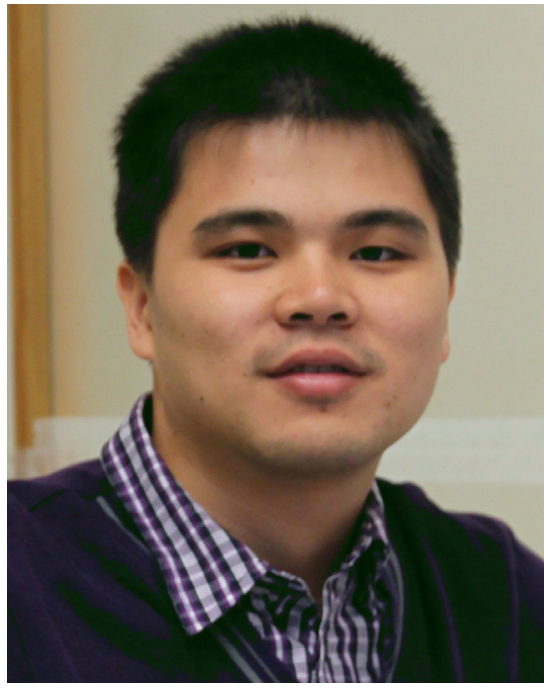

(b) Honglong Chen

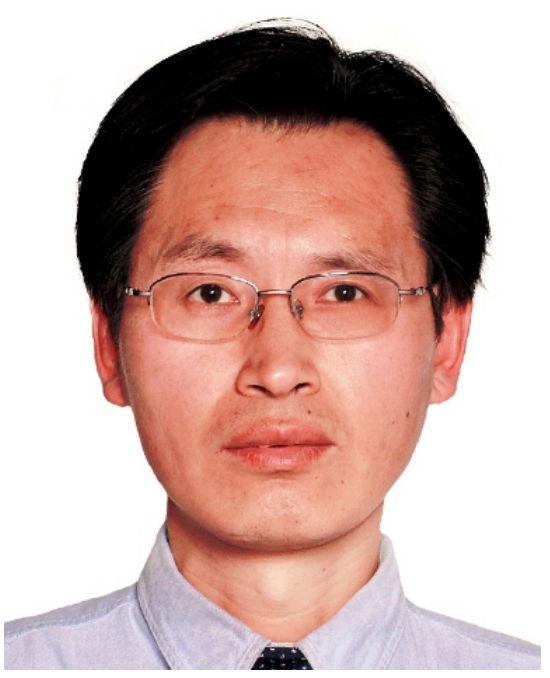

(e) Zhi Wang

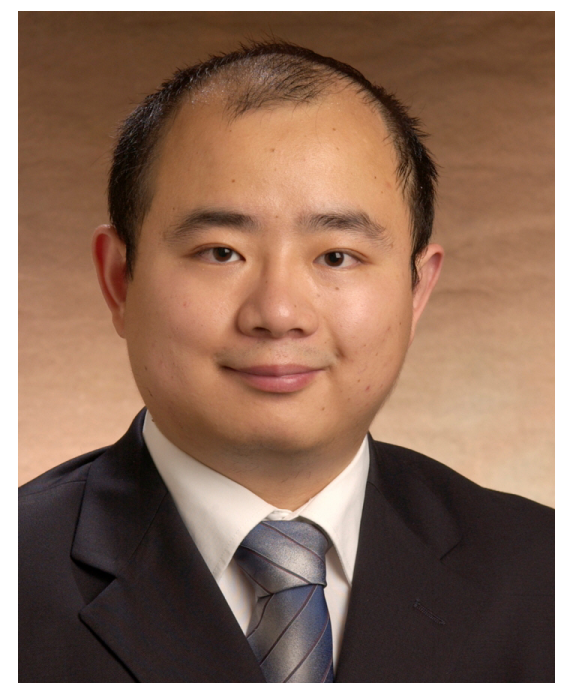

(c) Qing Cao

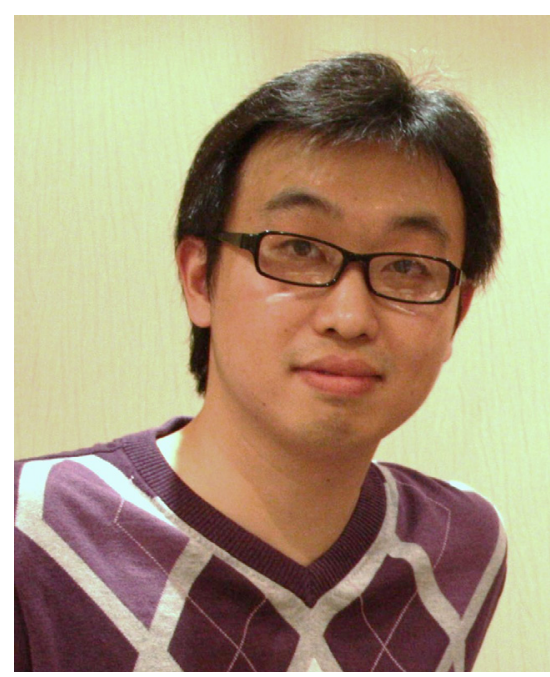

(f) Qian Wang

Fig. 1 